The Astrophysical Journal, 629:482-498, 2005 August 10

(C) 2005. The American Astronomical Society. All rights reserved. Printed in U.S.A.

\title{
BULK VELOCITIES, CHEMICAL COMPOSITION, AND IONIZATION STRUCTURE OF THE X-RAY SHOCKS IN WR 140 NEAR PERIASTRON AS REVEALED BY THE CHANDRA GRATINGS
}

\author{
A. M. T. Pollock ${ }^{1}$ \\ European Space Agency, XMM-Newton Science Operations Centre, European Space Astronomy Centre, \\ Apartado 50727, Villafranca del Castillo, 28080 Madrid, Spain; andy.pollock@esa.int \\ Michael F. CoRCORAN \\ Universities Space Research Association, 7501 Forbes Boulevard, Suite 206, Seabrook, MD 20706; \\ and Laboratory for High Energy Astrophysics, Goddard Space Flight Center, Greenbelt, MD 20771 \\ IAN R. STEVEnS \\ School of Physics and Astronomy, University of Birmingham, Birmingham B15 2TT, England \\ AND \\ P. M. Williams \\ Institute for Astronomy, Royal Observatory, Blackford Hill, Edinburgh EH9 3HJ, Scotland \\ Received 2004 April 7; accepted 2005 April 14
}

\begin{abstract}
The Wolf-Rayet WC7+O4-5 binary WR 140 went through the periastron passage of its 8 yr eccentric binary orbit in early 2001 as the two stars made their closest approach. Both stars have powerful supersonic stellar winds that crash into each other between the stars to produce X-rays. Chandra grating observations were made when the X-rays were at their peak, making WR 140 the brightest hot-star X-ray source in the sky and giving the opportunity to study the velocity profiles of lines, all of which were resolved and blueshifted before periastron. In the general context of shock physics, the measurements constrain the flow of hot gas and where different ions were made. The brightness of lines relative to the strong continuum in conjunction with plasma models gives interim abundance estimates for eight different elements in WC-type material including an $\mathrm{Ne} / \mathrm{S}$ ratio in good agreement with earlier long-wavelength measurements. The lower velocity widths of cool ions imply a plasma that was not in equilibrium, probably due to the collisionless nature of the shock transitions and the slow character of both the postshock energy exchange between ions and electrons and subsequent ionization. Electron heat conduction into fast-moving preshock gas was absent, probably suppressed by the magnetic field involved in WR 140's synchrotron emission. After periastron, the spectrum was weaker due mainly to absorption by cool Wolf-Rayet star material.
\end{abstract}

Subject headings: binaries: close — shock waves — stars: abundances — stars: individual (WR 140) stars: winds, outflows - stars: Wolf-Rayet - X-rays: stars

\section{INTRODUCTION}

There is no doubt that the brilliance of the X-rays from the hot-star binary system WR 140 is due to the interaction of the two winds of its component WC7 and O4-5 stars. The system has become the prototype colliding-wind binary (Williams et al. 1990) to which profitable attention may be turned to investigate ideas in several challenging areas of physics, made all the more interesting by the $7.94 \mathrm{yr}$ period and extreme eccentricity of its orbit. The giant IR outbursts that repeat soon after periastron passage, the most recent of which was imaged by Monnier et al. (2002), are widely supposed to be due to explosive dust formation in an environment apparently much too hostile for any such thing. The distinctive orbital modulation of the nonthermal radio flux (White \& Becker 1995) has also so far escaped any convincing explanation, although it is generally understood that centimeter radiation can only escape through the weaker $\mathrm{O}$ star wind. Marchenko et al. (2003) followed WR 140 between its periastron passages of 1993 and 2001 with an intensive campaign of optical spectroscopy and photometry and were able to confirm the high eccentricity of the system, $e=0.881 \pm 0.005$,

\footnotetext{
${ }^{1}$ Formerly at Computer \& Scientific Co. Ltd., 230 Graham Road, Sheffield S10 3GS, England.
}

and derive orbital elements for both stars including a period that matches the Williams et al. (1990) estimate based on the repeatability of the IR outbursts.

Despite the fascination of these questions, it is X-rays that are central, literally and figuratively, to WR 140 's colliding winds. The system is bright enough to have been the target for most X-ray observatories since its discovery in 1984 with EXOSAT (e.g., Pollock 1987; Williams et al. 1990; Zhekov \& Skinner 2000; Pollock et al. 2002). Between the stars, shock dissipation of the flow energy of the winds, both of which have terminal velocities $v_{\infty} \sim 3000 \mathrm{~km} \mathrm{~s}^{-1}$, gives expected ion temperatures of several kilo-electron volts near the stagnation point. As discussed below, the shocks are collisionless, so that attention must be given to the evolution of both the electron temperature and the ionization state of the postshock gas that together determine the lines and continuum of the emitted spectrum. There is no stationary gas in WR 140 . Continuity demands that all shocked material escapes the system within a time of $\left(D / v_{\infty}\right)$, where $D$ is the separation of the stars, which ranges from about $10^{5} \mathrm{~s}$ near periastron to $10^{6} \mathrm{~s}$ near apastron during which adiabatic cooling also takes place.

Relevant parameters of the WR 140 system that have been used here are given in Table 1. The expected interstellar column density, which is large enough to cause considerable soft X-ray 
TABLE 1

Relevant Parameters of the WR 140 WC7+O4-5 Binary System

\begin{tabular}{|c|c|}
\hline Parameter & Value \\
\hline Binary period $^{\mathrm{a}} P($ days $) \ldots \ldots$. & $2899.0 \pm 1.3$ \\
\hline Orbital eccentricity ${ }^{\mathrm{a}} e \ldots \ldots . . .$. & $0.881 \pm 0.005$ \\
\hline Orbital semimajor axis ${ }^{\mathrm{a}} a \sin i(\mathrm{AU})$ & $14.1 \pm 0.5$ \\
\hline Date of periastron passage ${ }^{\mathrm{a}} T_{0}$ (HJD) & $2446147.4 \pm 3.7$ \\
\hline 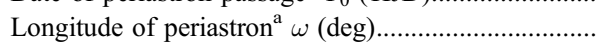 & $46.7 \pm 1.6$ \\
\hline Inclination $i(\mathrm{deg})$ & 60 \\
\hline 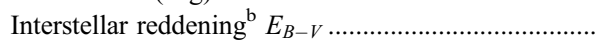 & $0.95 \pm 0.03$ \\
\hline Interstellar column density $N_{\mathrm{H}}\left(10^{21} \mathrm{~cm}^{-2}\right) \ldots \ldots \ldots \ldots \ldots$ & $5.9 \pm 0.6$ \\
\hline Distance $^{\mathrm{c}} d(\mathrm{kpc})$ & 1.1 \\
\hline
\end{tabular}

WC7

\begin{tabular}{|c|c|}
\hline Stellar radius ${ }^{\mathrm{d}} R_{*}\left(R_{\odot}\right) \ldots \ldots \ldots \ldots \ldots$ & 13 \\
\hline Mass-loss rate ${ }^{\mathrm{d}} \dot{M}_{*}\left(M_{\odot} \mathrm{yr}^{-1}\right) \ldots$ & $5.7 \times 10^{-5}$ \\
\hline Terminal velocity ${ }^{\mathrm{d}} v_{\infty}\left(\mathrm{km} \mathrm{s}^{-1}\right)$. & 2860 \\
\hline
\end{tabular}

O4-5

Stellar radius ${ }^{\mathrm{d}} R_{*}\left(R_{\odot}\right)$. 12

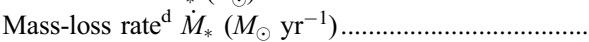

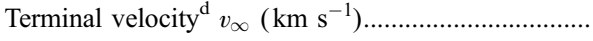

$1.8 \times 10^{-6}$

3200

\footnotetext{
a Marchenko et al. (2003).

b Morris et al. (1993).

c Van der Hucht (2001).

d Williams et al. (1990).
}

absorption, was calculated from $E_{B-V}$ using the Vuong et al. (2003) compilation value of $N_{\mathrm{H}} / A_{V}=(2.0 \pm 0.2) \times 10^{21} \mathrm{~cm}^{-2}$ assuming $R_{V}=A_{V} / E_{B-V}=3.1$. The system inclination is more difficult to determine, although observational constraints have been derived by Setia Gunawan et al. (2001), Marchenko et al. (2003), Varricatt et al. (2004), and Dougherty et al. (2005) that are consistent with our adopted value of $i=60^{\circ}$. The distance to WR 140 is also uncertain, and Dougherty et al. (2005) have recently argued for a value of $d=1.85 \pm 0.16 \mathrm{kpc}$ based on the resolution of the individual stars in the near-infrared by Monnier et al. (2004) coupled with analysis of resolved Very Long Baseline Array (VLBA) radio measurements.

The launch of the grating spectrometers aboard Chandra and $X M M-N e w t o n$ came at a particularly good time for those interested in WR 140 as, according to the Williams et al. (1990) ephemeris, it was due to go through its periastron passage early in 2001 when the X-rays are at their brightest and circumstellar absorption can be prominent. The high spectral resolution of these instruments, with their ability to resolve the Doppler structure of individual emission lines in winds as fast as those in WR 140, promised to be no less important for the study of the hightemperature plasma in colliding winds than it has proved for stellar coronae and supernova remnants (SNRs), for example. While visibility problems prevented any XMM-Newton observations, we were awarded two Chandra grating observations of WR 140 near the 2001 periastron passage through Director's Discretionary Time under the auspices of the XMEGA collaboration. ${ }^{2}$

\section{THE CHANDRA CAMPAIGN}

The timing of the two Chandra observations was very important. On the basis of previous EXOSAT, ROSAT, and ASCA observations (Pollock et al. 2002) interpreted using the Stevens et al. (1992) colliding spherical wind models, we expected the luminosity to increase steadily with little change of spectrum

\footnotetext{
${ }^{2}$ See http://lheawww.gsfc.nasa.gov/users/corcoran/xmega/xmega.html.
}

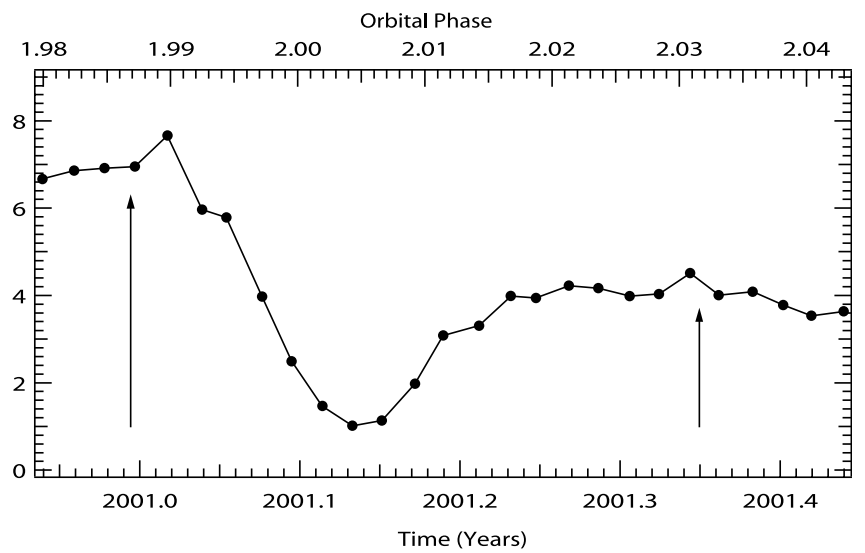

FIG. 1.-WR 140's RXTE 2-10 keV count rate during the first 6 months of the weekly monitoring campaign. Most of the low-level variability after the $\mathrm{X}$-ray minimum is caused by background variations. The dates of the Chandra observations are shown by the arrows.

before periastron, as the stars approach, until the sudden appearance in the line of sight of cool, unshocked absorbing WC material a few weeks before periastron. Given the contemporary uncertainties in the ephemeris, which amounted to about 50 days in the time of periastron passage, we requested the first exposure before the end of 2000 to ensure a bright spectrum with little or no circumstellar absorption with the second initially scheduled in 2001 March near the expected periastron in order to capture the absorption spectrum.

\subsection{Accompanying RXTE Observations}

At about the same time, we had also successfully proposed in Cycle 6 for $R X T E$ weekly monitoring to cover the period around periastron and beyond. Through the good offices of the $R X T E$ schedulers, these weekly proportional counter observations started early at the beginning of 2000 December and continued for 24 months. They will be described in detail elsewhere but were invaluable in defining the context of the Chandra grating spectra. The WR 140 RXTE count rate data shown in Figure 1 emphasize how fortunate the timing of the first observation proved to be, just over a week before X-ray maximum. The fast turnaround time of $R X T E$ data processing allowed us to monitor WR 140's condition in the weeks following the first observation. In particular, alarmed at the rapid development of the eclipse, we were able to postpone the second exposure until the flux had started to recover to give enough photons for Chandra's gratings to do their job.

\subsection{The Chandra Grating Observations of WR 140 near Periastron}

Table 2 reports details of the two observations including the binary phase, true anomaly, and stellar separation calculated according to the Marchenko et al. (2003) orbit. Although we expect to be able to refine knowledge of the orbit in the near future, so that the phases may alter slightly, there is no doubt that the observations took place a few weeks either side of periastron passage. Although the inclination is not well known, the Marchenko et al. (2003) orbit firmly defines via the true anomaly values the azimuthal orientation of the stars during the two observations. Figure 2 illustrates the approximate orientations of the binary system during the two observations to an Earth-bound observer looking from the bottom of the figure.

Chandra has two high-energy gratings, designated HEG and MEG, which are aligned so that the dispersed positive and negative orders form a shallow "X" in the focal plane on the set of six ACIS-S 
TABLE 2

The Two Chandra ACIS-S Grating Observations of WR 140

\begin{tabular}{|c|c|c|}
\hline Parameter & Observation 1 & Observation 2 \\
\hline ObsID ..... & 2337 & 2338 \\
\hline Sequence number.............. & 200124 & 200125 \\
\hline 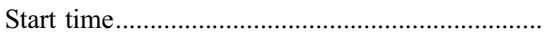 & 2000 Dec 29 10:13:28 & 2001 May 08 19:48:01 \\
\hline End time & 2000 Dec 29 23:38:48 & 2001 May 09 03:05:52 \\
\hline 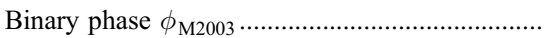 & 1.987 & 2.032 \\
\hline 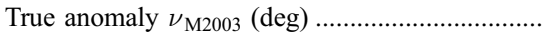 & 267.6 & 123.9 \\
\hline 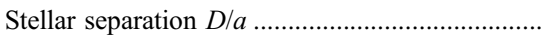 & 0.246 & 0.439 \\
\hline Exposure time $(\mathrm{s})$ & 45543 & 24524 \\
\hline 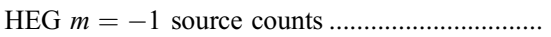 & 13417 & 1871 \\
\hline HEG $m=-1$ background counts ......................... & 72 & 19 \\
\hline 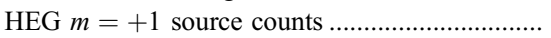 & 12555 & 1962 \\
\hline HEG $m=+1$ background counts ........................ & 60 & 18 \\
\hline 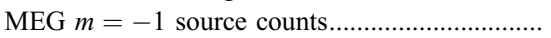 & 23718 & 2247 \\
\hline MEG $m=-1$ background counts ........................ & 115 & 28 \\
\hline MEG $m=+1$ source counts............................... & 26661 & 2993 \\
\hline MEG $m=+1$ background counts ...................... & 160 & 83 \\
\hline
\end{tabular}

CCDs. The HEG and MEG have FWHM resolutions of 12 and $23 \mathrm{~m} \AA$, respectively, and wavelength accuracies of 6 and $11 \mathrm{~m} \AA$ in absolute terms and half those values in relative terms. The capabilities of the Chandra instruments are described by Weisskopf et al. (2002) with greater technical detail available in the Chandra Proposers' Observatory Guide. ${ }^{3}$ The analysis we report here is based on the standard pipeline products and subsequent analysis with versions of $\mathrm{CIAO}^{4}$ between version 2.1 and version 3.0.2, with corresponding calibration data up to CALDB 2.25, and XSPEC ${ }^{5}$

\footnotetext{
3 Available at http://cxc.harvard.edu/proposer/POG/index.html.

${ }^{4}$ See http://cxc.harvard.edu/ciao.

5 See http://xspec.gsfc.nasa.gov/docs/xanadu/xspec/index.html.
}

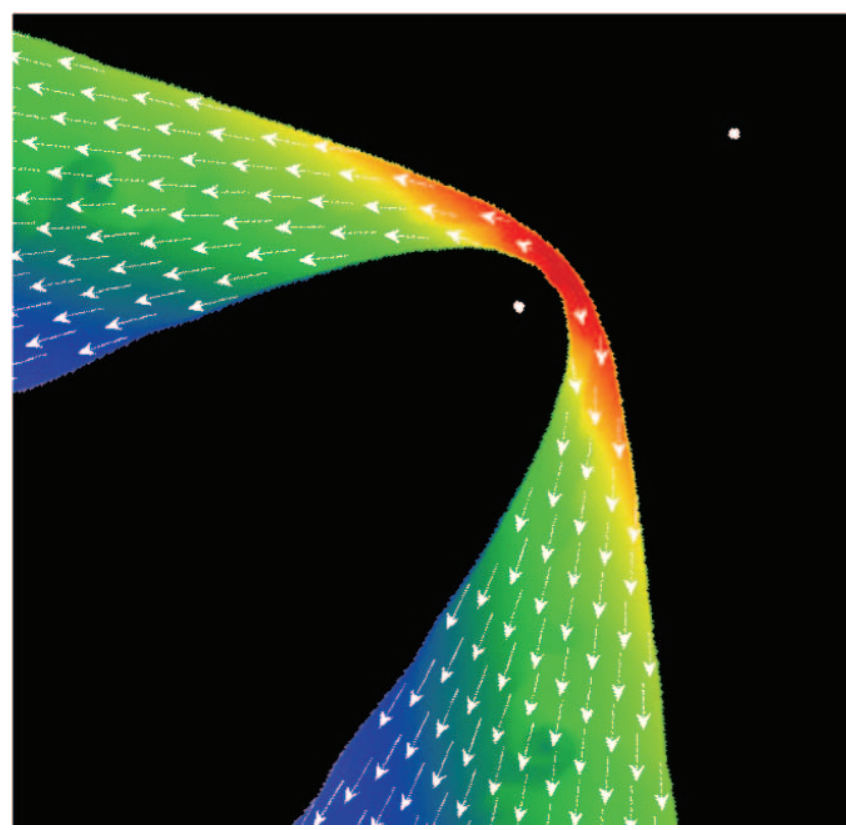

up to version 11.1. Laboratory wavelengths have been taken mainly from the APED (Smith et al. 2001) and CHIANTI (Young et al. 2003) lists. We have calculated parameters from a simultaneous treatment of HEG and MEG $m= \pm 1$ order spectra with custom-generated RMFs by using the likelihood $C$-statistic without rebinning in order to preserve all spectral information in the data. We have made use of the $C$-statistic below both in spectral parameter estimation and for quantitative comparison of alternative models. The spectra were limited, as expected on the basis of the star's visual color excess, by interstellar absorption at long wavelengths and thus cover the range 1-20 $\AA$ unless further restricted by circumstellar absorption. The images show a zeroth-order undispersed image of WR 140 at the center of the

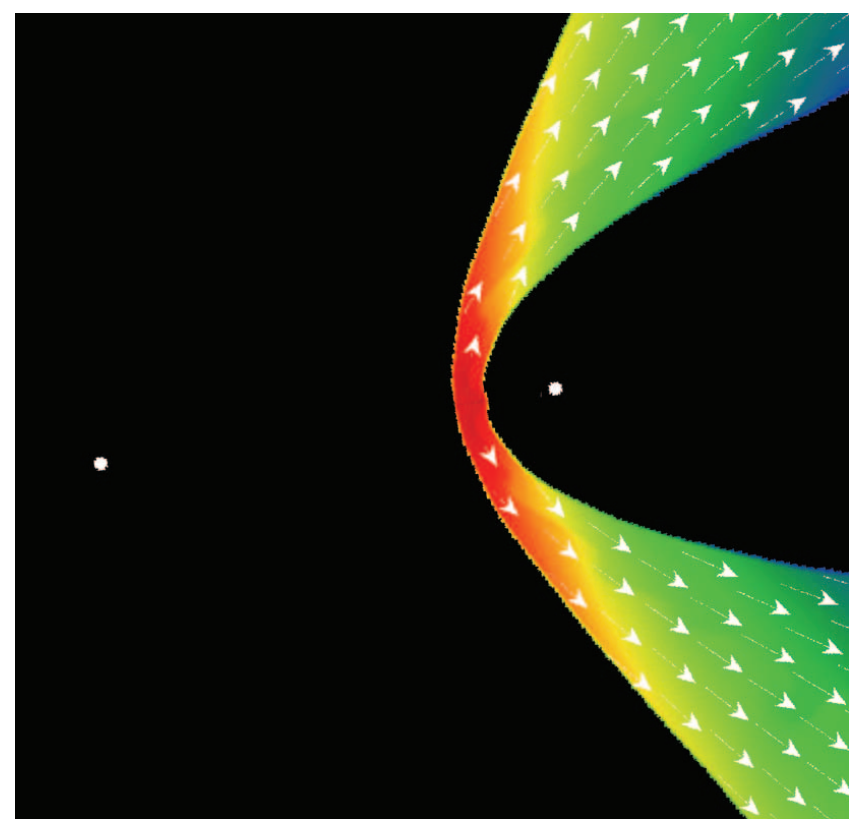

FIG. 2.-Approximate aspects of X-ray-bright material in WR 140 to an Earth-bound satellite at the bottom of the figure of the Chandra observation before periastron on 2000 December 29 on the left and after periastron on 2001 May 8 on the right. These are sections through the orbital plane of the binary system of numerical models using the Stevens et al. (1992) parameters for WR 140. The positions of the stars are shown by the small white circles; the Wolf-Rayet component with its more powerful wind faces the convex shock surface. The Wolf-Rayet and O star winds remain cool unless they encounter the shock and are thus black or invisible to the X-ray observer. Before periastron, much X-ray material is flowing directly toward the line of sight. Shortly afterward, as confirmed by Fig. 1, cool opaque WolfRayet material will have swung into view and still be there after periastron to obscure the view of hot material then expected to be flowing largely sideways. 


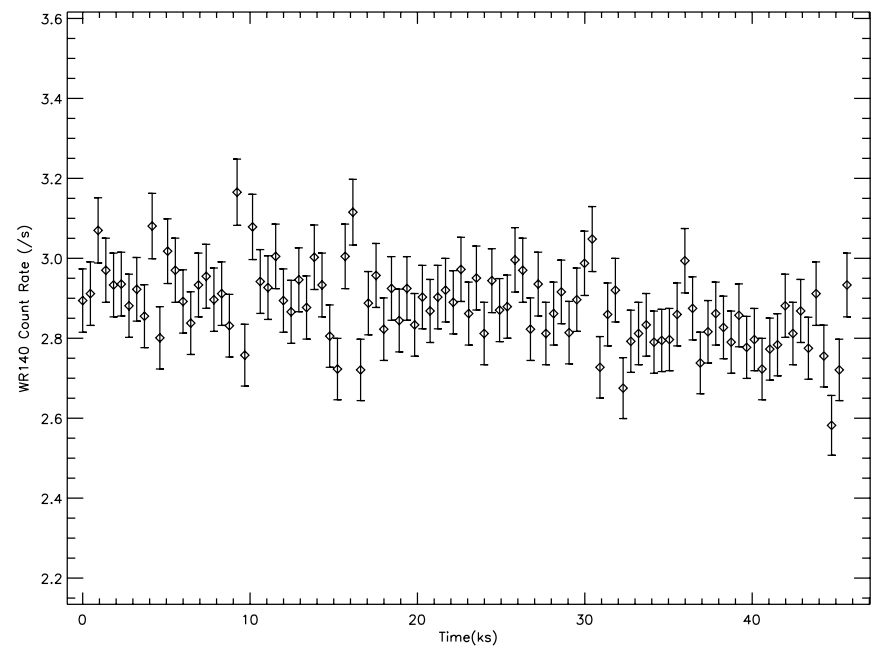

FIG. 3.-WR 140's total Chandra HETG 1-20 A count rate with $1 \sigma$ Poisson counting errors during the pre-periastron observation of 2000 December 29 , dividing the elapsed time into 100 equal intervals of $461 \mathrm{~s}$. In order to maximize statistics, all events with a valid order were selected, excluding the piled-up zeroth order. The background was weak enough to be ignored.

dispersed spectra. No other sources were detected by the standard processing anywhere in either field.

We did not use the background estimates supplied with the spectra because, although weak, they contained more counts from WR 140 itself than from any other cosmic, particle, or instrumental components. Inspection of the cross-dispersion distribution of data of each grating arm showed indeed that the default selection regions used for generation of the background spectra contain the far wings of events from WR 140. Using instead background selec- tion regions in the range $0.002 \leq\left|\operatorname{tg} \_d\right| \leq 0.004$, where tg_d is the event file cross-dispersion angle, showed that the local backgrounds were flat on a scale of a few arcseconds and, when extrapolated into the central \pm 2 ". 4 source selection region, gave the negligibly small levels reported with their $1 \sigma$ Poissonian errors in Table 2. WR 140 lies in the region of diffuse emission associated with the Cygnus superbubble and appears clearly at $(l, b)=$ $\left(80^{\circ} .93,4.18\right)$ in the ROSATM- and H-band plots in Figures 3 and 4 of Uyaniker et al. (2001). Although Zhekov \& Skinner (2000) needed to take explicit account of this background in their $A S C A$ analysis of WR 140, the Chandra grating diffraction and order selection criteria combine the narrow instrumental point-spread function with the explicit position of WR 140 to exclude very effectively superbubble emission from the star's dispersed spectra.

\subsection{The Bright Pre-Periastron Observation of 2000 December 29}

During the $12.65 \mathrm{hr}$ exposure obtained near maximum light before periastron in late 2000 December, just over a week before the onset of the X-ray eclipse, WR 140 became the brightest hotstar X-ray source to be observed by Chandra or any other instrument, with MEG first-order count rates of over 0.5 counts s ${ }^{-1}$. There is good evidence that it was variable, decreasing by about $10 \%$ during the observation. Figure 3 shows the count rate of all available dispersed events with an assigned order but excluding the piled-up zeroth order. For the 100 bins, $\chi_{99}^{2}=149.7$ for a constant rate leading to a rejection of this hypothesis at the $99.9 \%$ level. The background count rate calculated from the rest of the field showed no variability. The only worthwhile comparison with another X-ray observation of a binary Wolf-Rayet star is with the Maeda et al. (1999) ASCA data of V444 Cygni in which few-hour variability was also observed.

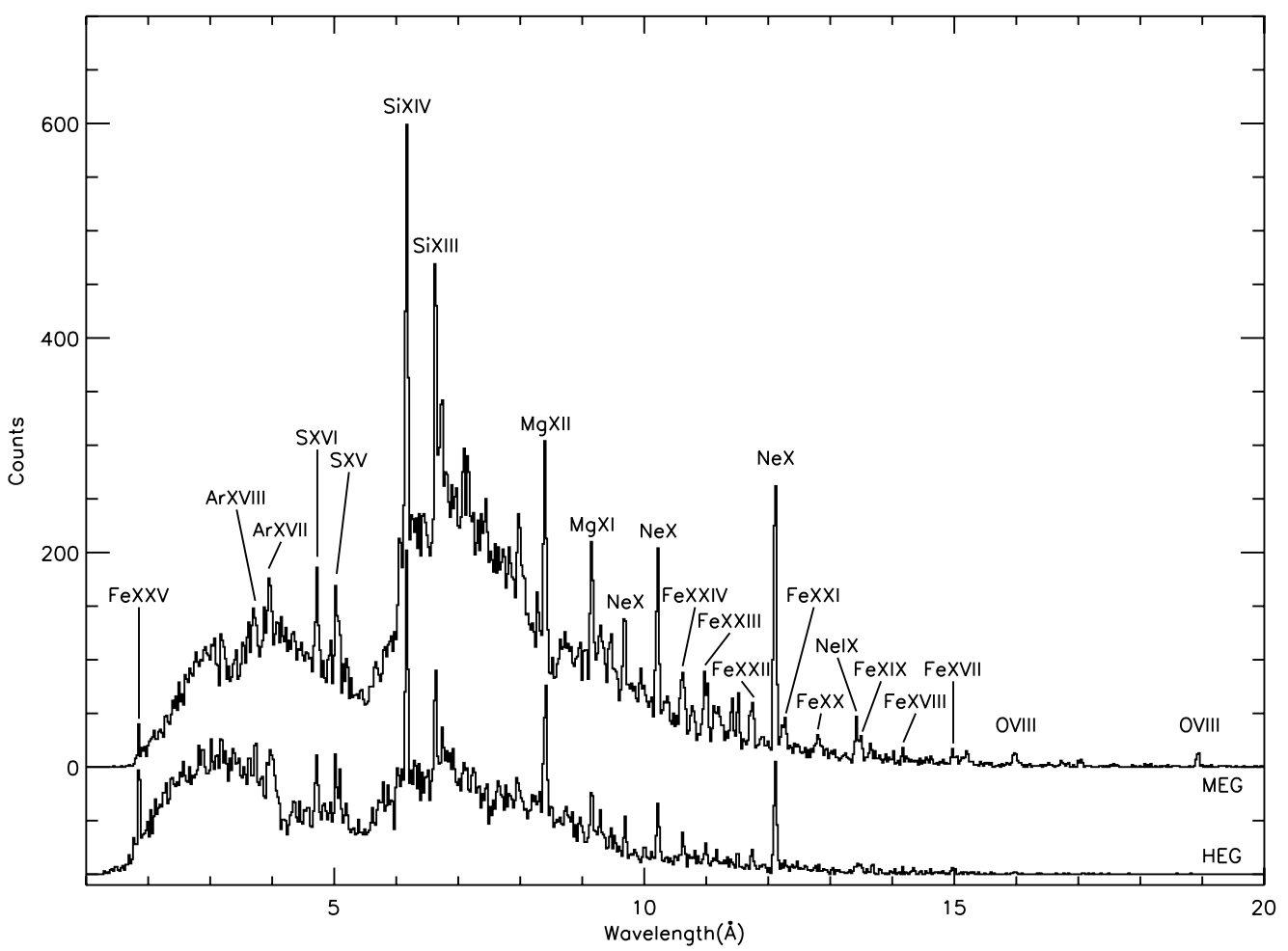

FIG. 4.-WR 140's Chandra first-order HEG and MEG spectra during the pre-periastron observation of 2000 December 29. Positive and negative orders have been added together and the spectra rebinned to emphasize the overall structure. The weaker HEG spectrum has been moved down by 100 counts. Above the strong continuum, the most prominent lines are Si XIV $\lambda 6.180, \operatorname{Si}$ XIII $\lambda 6.648, \operatorname{Mg}$ XII $\lambda 8.419$, and $\mathrm{Ne}$ x $\lambda \lambda 10.239,12.132$. Despite the decreasing effective area and the substantial effects of absorption at long wavelengths, there are clear detections as far as the principal Fe xvII lines near 15.015 $\AA$ and $\mathrm{O}$ VIII $\lambda \lambda 16.005,18.967$. A complete list of detected lines is given in Tables 3 and 4 . 
We have ignored this variability in the spectral analysis by summing all the available data to give the overall HEG and MEG spectra shown in Figure 4. The spectra show prominent emission lines, some of which are identified in the figure, and a strong continuum along with the substantial effects of interstellar absorption at long wavelengths expected from the star's reddening. The decrease in counts at long wavelengths is also partly caused by wavelength dependence of the effective area, which peaks in the MEG near $7 \AA$ and had fallen by about a factor of 3 by $13 \AA$.

\subsubsection{The Pre-Periastron Emission Lines}

Above the continuum, the spectrum showed a mixture of $\mathrm{H}$-like and He-like emission lines from abundant elements $\mathrm{O}$, $\mathrm{Ne}, \mathrm{Mg}, \mathrm{Si}$, and $\mathrm{S}$ along with a variety of Fe lines from different ionization stages including He-like Fe Xxv and all the L-shell ions between Fe XVII and Fe XxIV. Ca and Fe XXVI were not detected. Si had the strongest lines, and despite the interstellar absorption, $\mathrm{Ne} x$ was strong with $\operatorname{Ly} \alpha, \operatorname{Ly} \beta, \operatorname{Ly} \gamma$, and $\operatorname{Ly} \delta$ all prominent. Even near 19 and $16 \AA$ the handful of detected photons were clearly from $\mathrm{O}$ VIII Ly $\alpha$ and $\operatorname{Ly} \beta$, respectively. Despite the detection, in particular, of the Ne $\mathrm{x}$ lines up to $\mathrm{Ly} \delta$, there was no evidence of a radiative recombination continuum in this or any other ion.

Most of the lines were obviously blueshifted with roughly equal velocities near $-600 \mathrm{~km} \mathrm{~s}^{-1}$ and were significantly wider than the instrumental response. The wavelength shifts were not constant but scaled with wavelength and were consistent between HEG and MEG and between negative and positive orders, so that they do not result from calibration errors in the wavelength scale. The excess width is confirmed by the comparison shown in Figure 5 with the width of zeroth-order frame-transfer streak as described on page 162 of the Chandra POG version 5.0. Compared with narrow lines with widths fixed at the instrumental resolution, the best-fit broad-line models have improved $C$-statistic values of 22.4 for O vIII, 438.5 for $\mathrm{Ne}$ x, 220.2 for $\mathrm{Mg}$ XI, 217.9 for Si XIV, and 171.9 for S XVI.

We first estimated the observed fluxes and a mean velocity shift and width for each ion species by isolating the relevant set of lines and about half an angstrom's width of adjacent continuum and fitting Gaussian profiles above a polynomial continuum simultaneously to all four HEG $m= \pm 1$ and MEG $m=$ \pm 1 spectra wherever possible; the wavelengths and widths of subsidiary lines were constrained to have the same Doppler shifts as the nominated reference lines of the stronger component of $\mathrm{H}$-like Ly $\alpha$ or the resonance component of the He-like triplet. The fluxes of the weaker component of the H-like doublet were fixed at half of the stronger, while the fluxes of the fir components of the He-like triplets were all free parameters. Because we made use of the custom-made response files mentioned above that incorporate the HEG and MEG instrumental widths of 12 and $23 \mathrm{~m} \AA$, respectively, the fitted widths reflect the velocity dispersion of WR 140's lines. For the elements $\mathrm{O}, \mathrm{Ne}, \mathrm{Mg}, \mathrm{Al}$, Si, S, and Ar Table 3 reports the wavelength, width, and flux of the emission lines identified before periastron in WR 140's spectrum. The lines from the various ionization stages of $\mathrm{Fe}$ are reported separately in Table 4 . In both tables, electronic configurations were taken from APED and CHIANTI. Symmetric Gaussian models are good fits to the data. Despite the similar blueshifts across the ensemble of lines, the velocity widths do not agree but increase systematically with excitation by a factor of 2 or 3 from less than 1000 to more than $2000 \mathrm{~km} \mathrm{~s}^{-1}$. $\mathrm{Ne} x$, for example, is seen from gas velocities in the range about $-1600 \leq v_{\mathrm{Nex}}\left(\mathrm{km} \mathrm{s}^{-1}\right) \leq+500$, while Si XIV comes from a broader range of about $-2000 \leq v_{\mathrm{Si} \text { XIV }}\left(\mathrm{km} \mathrm{s}^{-1}\right) \leq+1000$ as shown in Figure 6. The ion Doppler velocities calculated from
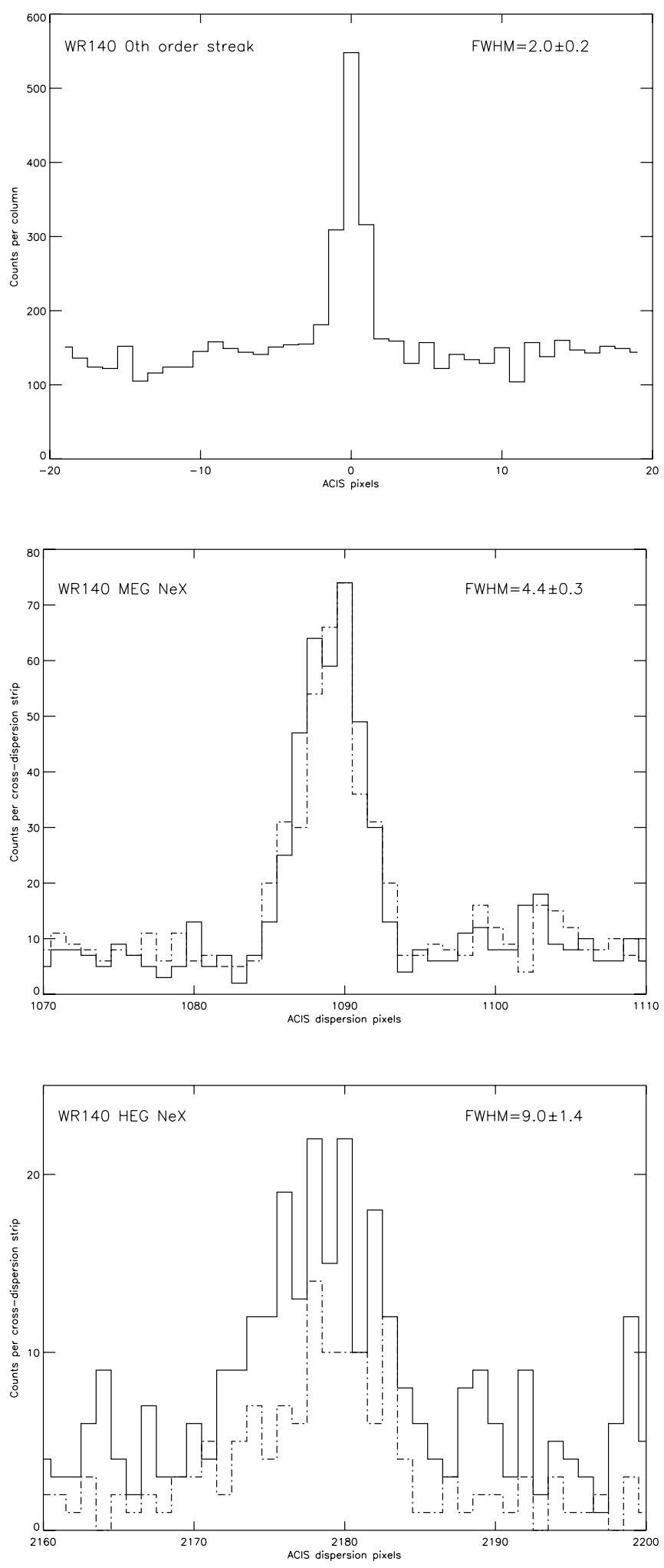

FIG. 5.-Observed distributions in pixel coordinates of the zeroth-order frame-transfer streak that is due to instrumental broadening in comparison with the superimposed MEG and $\mathrm{HEG} \pm 1$ orders along the dispersion direction of the Ne $\mathrm{x}$ Ly $\alpha$ line. The solid line shows $m=-1$; the dashed line shows $m=+1$. The reported FWHM are in ACIS-S pixel units. The narrowness of the streak and the difference between the MEG and HEG lines confirm that the line was resolved by the Chandra grating instruments. 
TABLE 3

WR 140's H-like and He-like Emission Lines before Periastron

\begin{tabular}{|c|c|c|c|c|c|c|}
\hline Ion & Line & Transition & $\begin{array}{l}\lambda_{v=0} \\
(\AA)\end{array}$ & $\begin{array}{c}\lambda_{\mathrm{WR} 140} \\
(\AA)\end{array}$ & $\begin{array}{c}\text { FWHM } \\
(\mathrm{m} \AA)\end{array}$ & $\begin{array}{c}\text { Flux } \\
\left(10^{-5} \mathrm{~cm}^{-2} \mathrm{~s}^{-1}\right)\end{array}$ \\
\hline 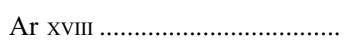 & Ly $\alpha$ & $2 p^{2} P \rightarrow 1 s^{2} S$ & 3.7311 & $3.7261 \pm 0.0051$ & $18.0 \pm 13.0$ & $1.85 \pm 0.61$ \\
\hline \multirow[t]{3}{*}{ 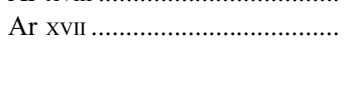 } & $r$ & $1 s 2 p^{1} P \rightarrow 1 s^{2}{ }^{1} S$ & 3.9488 & $3.9417 \pm 0.0067$ & $40.3 \pm 13.8$ & $3.54 \pm 0.83$ \\
\hline & $i$ & $1 s 2 p{ }^{3} P \rightarrow 1 s^{2}{ }^{1} S$ & 3.9656 & 3.9620 & $\ldots$ & $0.00 \pm 0.84$ \\
\hline & $f$ & $1 s 2 p^{3} S \rightarrow 1 s^{2}{ }^{1} S$ & 3.9939 & 3.9867 & $\cdots$ & $2.99 \pm 0.80$ \\
\hline \multirow[t]{2}{*}{ 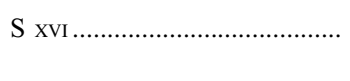 } & Ly $\alpha$ & $2 p^{2} P \rightarrow 1 s^{2} S$ & 4.7274 & $4.7184 \pm 0.0025$ & $40.0 \pm 6.2$ & $10.18 \pm 0.98$ \\
\hline & $\operatorname{Ly} \beta$ & $3 p^{2} P \rightarrow 1 s^{2} S$ & 3.9908 & 3.9834 & $\ldots$ & $0.00 \pm 0.75$ \\
\hline \multirow[t]{3}{*}{$\mathrm{S} x \mathrm{n}$} & $r$ & $1 s 2 p{ }^{1} P \rightarrow 1 s^{2}{ }^{1} S$ & 5.0387 & $5.0254 \pm 0.0018$ & $27.2 \pm 4.2$ & $8.59 \pm 0.92$ \\
\hline & $i$ & $1 s 2 p^{3} P \rightarrow 1 s^{2}{ }^{1} S$ & 5.0665 & 5.0533 & $\ldots$ & $1.92 \pm 0.82$ \\
\hline & $f$ & $1 s 2 p{ }^{3} S \rightarrow 1 s^{2}{ }^{1} S$ & 5.1015 & 5.0882 & $\ldots$ & $6.42 \pm 0.87$ \\
\hline \multirow[t]{3}{*}{ Si XIV } & Ly $\alpha$ & $2 p^{2} P \rightarrow 1 s^{2} S$ & 6.1804 & $6.1668 \pm 0.0008$ & $34.2 \pm 1.9$ & $16.34 \pm 0.63$ \\
\hline & $\operatorname{Ly} \beta$ & $3 p^{2} P \rightarrow 1 s^{2} S$ & 5.2168 & 5.2054 & $\ldots$ & $3.97 \pm 0.84$ \\
\hline & $\mathrm{Ly} \gamma$ & $4 p^{2} P \rightarrow 1 s^{2} S$ & 4.9467 & 4.9358 & $\ldots$ & $2.20 \pm 0.52$ \\
\hline \multirow[t]{3}{*}{ Si XIII } & $r$ & $1 s 2 p^{1} P \rightarrow 1 s^{2}{ }^{1} S$ & 6.6480 & $6.6343 \pm 0.0011$ & $32.3 \pm 2.3$ & $9.95 \pm 0.53$ \\
\hline & $i$ & $1 s 2 p{ }^{3} P \rightarrow 1 s^{2}{ }^{1} S$ & 6.6883 & 6.6748 & $\ldots$ & $2.13 \pm 0.44$ \\
\hline & $f$ & $1 s 2 p{ }^{3} S \rightarrow 1 s^{2}{ }^{1} S$ & 6.7404 & 6.7268 & $\cdots$ & $5.07 \pm 0.46$ \\
\hline 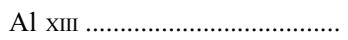 & Ly $\alpha$ & $2 p^{2} P \rightarrow 1 s^{2} S$ & 7.1709 & $7.1559 \pm 0.0048$ & $34.1 \pm 10.5$ & $1.71 \pm 0.35$ \\
\hline \multirow[t]{2}{*}{ 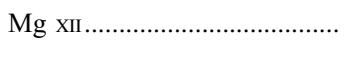 } & Ly $\alpha$ & $2 p^{2} P \rightarrow 1 s^{2} S$ & 8.4192 & $8.4008 \pm 0.0014$ & $49.6 \pm 2.9$ & $7.63 \pm 0.39$ \\
\hline & $\operatorname{Ly} \beta$ & $3 p^{2} P \rightarrow 1 s^{2} S$ & 7.1058 & 7.0911 & $\ldots$ & $0.91 \pm 0.25$ \\
\hline \multirow[t]{3}{*}{ 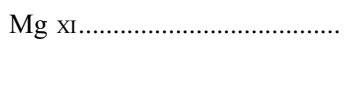 } & $r$ & $1 s 2 p{ }^{1} P \rightarrow 1 s^{2}{ }^{1} S$ & 9.1690 & $9.1516 \pm 0.0019$ & $38.0 \pm 3.9$ & $4.54 \pm 0.40$ \\
\hline & $i$ & $1 s 2 p{ }^{3} P \rightarrow 1 s^{2}{ }^{1} S$ & 9.2312 & 9.2134 & $\ldots$ & $0.22 \pm 0.31$ \\
\hline & $f$ & $1 s 2 p{ }^{3} S \rightarrow 1 s^{2}{ }^{1} S$ & 9.3143 & 9.2963 & $\ldots$ & $1.86 \pm 0.34$ \\
\hline \multirow[t]{4}{*}{$\mathrm{Ne} x$} & Ly $\alpha$ & $2 p^{2} P \rightarrow 1 s^{2} S$ & 12.1321 & $12.1071 \pm 0.0007$ & $41.0 \pm 1.6$ & $27.37 \pm 1.04$ \\
\hline & $\operatorname{Ly} \beta$ & $3 p^{2} P \rightarrow 1 s^{2} S$ & 10.2385 & 10.2174 & $\ldots$ & $7.33 \pm 0.48$ \\
\hline & $\mathrm{Ly} \gamma$ & $4 p^{2} P \rightarrow 1 s^{2} S$ & 9.7080 & 9.6880 & $\ldots$ & $3.26 \pm 0.36$ \\
\hline & Ly $\delta$ & $5 p^{2} P \rightarrow 1 s^{2} S$ & 9.4807 & 9.4612 & $\ldots$ & $2.09 \pm 0.32$ \\
\hline \multirow[t]{4}{*}{ 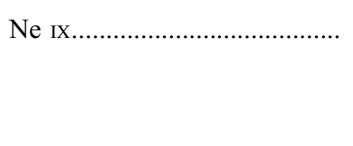 } & $r$ & $1 s 2 p{ }^{1} P \rightarrow 1 s^{2}{ }^{1} S$ & 13.4470 & $13.4187 \pm 0.0032$ & $43.6 \pm 7.2$ & $4.34 \pm 0.63$ \\
\hline & $i$ & $1 s 2 p{ }^{3} P \rightarrow 1 s^{2}{ }^{1} S$ & 13.5529 & 13.5244 & $\ldots$ & $0.00 \pm 0.39$ \\
\hline & $f$ & $1 s 2 p^{3} S \rightarrow 1 s^{2}{ }^{1} S$ & 13.6987 & 13.6699 & $\cdots$ & $2.24 \pm 0.51$ \\
\hline & $\mathrm{He} \beta$ & $1 s 3 p{ }^{1} P \rightarrow 1 s^{2}{ }^{1} S$ & 11.5467 & 11.5191 & $\cdots$ & $3.03 \pm 0.41$ \\
\hline \multirow{3}{*}{ 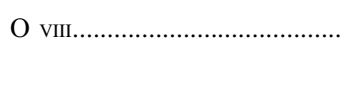 } & Ly $\alpha$ & $2 p^{2} P \rightarrow 1 s^{2} S$ & 18.9671 & $18.9264 \pm 0.0044$ & $53.6 \pm 9.3$ & $6.43 \pm 1.24$ \\
\hline & $\operatorname{Ly} \beta$ & $3 p^{2} P \rightarrow 1 s^{2} S$ & 16.0050 & 15.9712 & $\ldots$ & $2.18 \pm 0.63$ \\
\hline & $\mathrm{Ly} \gamma$ & $4 p^{2} P \rightarrow 1 s^{2} S$ & 15.1760 & 15.1477 & $\ldots$ & $1.77 \pm 0.50$ \\
\hline
\end{tabular}

Notes. - The H-like fluxes are the sum of the doublet a and $\mathrm{b}$ components assuming $f_{\mathrm{a}}=2 f_{\mathrm{b}}$. A single reference wavelength for each ion was treated as a free parameter as shown by the reported errors; other wavelengths were fixed according to the laboratory wavelength ratios. Parameters that were free have an associated error; those that were constrained do not.

the line profiles are shown in Table 5 and plotted against ionization potential in Figure 7.

\subsubsection{The He-like Triplets}

Among the He-like fir triplets often used as density and temperature diagnostics, strong resonance and forbidden lines were observed from $\mathrm{Ne}$ IX, Mg XI, Si XIII, and S XV with the intercombination lines weak or absent as shown in Figure 8 . The Chandra resolution, coupled with the wavelength constraints imposed, is good enough to conclude that emission observed between the Ne IX $r$ and $f$ components was dominated by the strongest lines of Fe XIX rather than the intercombination line as also observed in the Sun by Wolfson et al. (1983) and more recently in several cool stars by Ness et al. (2002). For Fe xxv, the separation of the He-like triplet is comparable to the velocity dispersion, making it impossible to resolve the individual fir components, so that the velocity width in Table 5 was calculated after fixing the blueshift at the ensemble average of the other lines.

WR 140 falls in a category of stars with $\gamma$ Velorum (Skinner et al. 2001) and $\eta$ Carinae (Corcoran et al. 2001), whose weak or absent intercombination lines contrast with the group exemplified by the prominent $\mathrm{O}$ stars $\zeta$ Puppis, $\zeta$ Orionis, and $\delta$ Orionis (e.g., Miller et al. 2002). The O star intercombination lines are much stronger than the forbidden lines probably due to effects of photospheric UV radiation on X-ray production deep in the wind. It is tempting to identify these two groups of X-ray sources with colliding-wind binary systems and single stars, respectively. In WR 140 and $\eta$ Carinae the $R=f / i$ ratios for all the elements observed look as though they exceed even the collisional equilibrium low-density upper limits $R 0$ given, for example, by Pradhan (1982) or Porquet et al. (2001). The observed line ratios for WR 140 are shown in Table 6 along with representative theoretical values of $R 0$. The choice of $R 0$ was not completely straightforward given, as discussed below, that collisional equilibrium does not obviously apply. However, the values do not change much over a large range of physical conditions. While in WR 140 the statistical significance of the unexpectedly high values of $R$ needs confirmation, in $\eta$ Carinae (Corcoran et al. 2001), whose lines are narrower and therefore easier to resolve, the effect is more clear-cut with only upper limits for the intercombination lines. Possible origins of this behavior are discussed below.

\subsubsection{Iron in the Spectrum}

The relative simplicity of the light-element $\mathrm{H}$-like and He-like lines makes them reasonably straightforward to identify. The complex iron L-shell emission that dominates the spectrum between 10 and $18 \AA$ of many coronal stellar sources, including the Sun (e.g., McKenzie et al. 1980) and Capella (e.g., Behar et al. 
TABLE 4

WR 140's Fe Emission Lines before Periastron

\begin{tabular}{|c|c|c|c|c|c|c|}
\hline Ion & Line & Transition & $\begin{array}{l}\lambda_{v=0} \\
(\AA)\end{array}$ & $\begin{array}{c}\lambda_{\mathrm{WR} 140} \\
(\AA)\end{array}$ & $\begin{array}{c}\text { FWHM } \\
(\mathrm{m} \AA)\end{array}$ & $\begin{array}{c}\text { Flux } \\
\left(10^{-5} \mathrm{~cm}^{-2} \mathrm{~s}^{-1}\right)\end{array}$ \\
\hline \multirow[t]{3}{*}{$\mathrm{Fe} x \mathrm{xv}$} & $r$ & $1 s 2 p{ }^{1} P \rightarrow 1 s^{2}{ }^{1} S$ & 1.850 & $1.8505 \pm 0.0013$ & $18.9 \pm 3.7$ & $15.05 \pm 1.67$ \\
\hline & $i$ & $1 s 2 p{ }^{3} P \rightarrow 1 s^{2}{ }^{1} S$ & 1.859 & 1.8594 & & $0.00 \pm 1.46$ \\
\hline & $f$ & $1 s 2 p{ }^{3} S \rightarrow 1 s^{2}{ }^{1} S$ & 1.868 & 1.8681 & & $1.38 \pm 1.15$ \\
\hline \multirow[t]{8}{*}{ Fe xxIV } & & $3 p^{2} P_{3 / 2} \rightarrow 2 s^{2} S_{1 / 2}$ & 10.619 & $10.6055 \pm 0.0024$ & $42.8 \pm 5.3$ & $4.82 \pm 0.31$ \\
\hline & & $3 p^{2} P_{1 / 2} \rightarrow 2 s^{2} S_{1 / 2}$ & 10.663 & 10.6494 & & 1.61 \\
\hline & & $3 d^{2} D_{3 / 2} \rightarrow 2 p^{2} P_{1 / 2}$ & 11.029 & 11.0150 & & $1.18 \pm 0.38$ \\
\hline & & $3 d^{2} D_{5 / 2} \rightarrow 2 p^{2} P_{3 / 2}$ & 11.171 & 11.1567 & & $1.72 \pm 0.44$ \\
\hline & & $3 s^{2} S_{1 / 2} \rightarrow 2 p{ }^{2} P_{1 / 2}$ & 11.261 & 11.2463 & & $0.82 \pm 0.34$ \\
\hline & & $3 s^{2} S_{1 / 2} \rightarrow 2 p{ }^{2} P_{3 / 2}$ & 11.426 & 11.4118 & & $2.32 \pm 0.41$ \\
\hline & & $4 d^{2} D \rightarrow 2 p^{2} P$ & 8.316 & 8.2985 & & $1.44 \pm 0.35$ \\
\hline & & $4 p^{2} P \rightarrow 2 s^{2} S$ & 7.984 & 7.9662 & & $1.27 \pm 0.22$ \\
\hline \multirow[t]{4}{*}{ 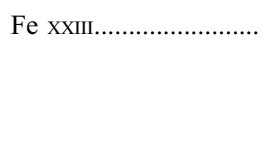 } & & $2 s 3 d^{1} D_{2} \rightarrow 2 s 2 p{ }^{1} P_{1}$ & 11.736 & $11.7221 \pm 0.0028$ & $37.7 \pm 7.3$ & $2.96 \pm 0.47$ \\
\hline & & $2 s 3 p^{1} P_{1} \rightarrow 2 s^{2}{ }^{1} S_{0}$ & 10.981 & 10.9749 & & $2.29 \pm 0.28$ \\
\hline & & $2 s 3 p^{3} P_{1} \rightarrow 2 s^{2}{ }^{1} S_{0}$ & 11.019 & 11.0060 & & 1.52 \\
\hline & & $2 s 3 s{ }^{1} S_{0} \rightarrow 2 s 2 p{ }^{1} P_{1}$ & 12.161 & 12.1687 & & $0.41 \pm 0.38$ \\
\hline Fe XXII ............. & & $3 d^{2} D_{3 / 2} \rightarrow 2 p^{2} P_{1 / 2}$ & 11.770 & 11.7561 & & $2.11 \pm 0.42$ \\
\hline \multirow{4}{*}{ 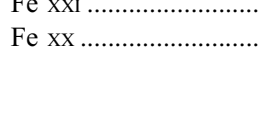 } & & $2 p 3 d d^{3} D_{1} \rightarrow 2 p^{2}{ }^{3} P_{0}$ & 12.285 & 12.2739 & & $1.66 \pm 0.45$ \\
\hline & & $2 p^{2} 3 d^{4} P_{1 / 2} \rightarrow 2 p^{3}{ }^{4} S_{3 / 2}$ & 12.824 & $12.7791 \pm 0.0071$ & & $0.79 \pm 0.15$ \\
\hline & & $2 p^{2} 3 d{ }^{4} P_{3 / 2} \rightarrow 2 p^{3}{ }^{4} S_{3 / 2}$ & 12.846 & 12.8010 & & 0.79 \\
\hline & & $2 p^{2} 3 d{ }^{4} P_{5 / 2} \rightarrow 2 p^{3}{ }^{4} S_{3 / 2}$ & 12.864 & 12.8189 & & 0.79 \\
\hline \multirow[t]{4}{*}{ Fe XIX } & & $2 p^{3} 3 d^{3} D_{3} \rightarrow 2 p^{4}{ }^{3} P_{2}$ & 13.518 & $13.4976 \pm 0.0050$ & $38.8 \pm 12.1$ & $2.33 \pm 0.37$ \\
\hline & & $2 p^{3} 3 d^{3} S_{1} \rightarrow 2 p^{4}{ }^{3} P_{2}$ & 13.462 & 13.4408 & & 0.96 \\
\hline & & $2 p^{3} 3 d^{3} D_{2} \rightarrow 2 p^{4}{ }^{3} P_{2}$ & 13.497 & 13.4758 & & 0.72 \\
\hline & & $2 p^{3} 3 d^{3} F_{3} \rightarrow 2 p^{4}{ }^{3} P_{2}$ & 13.645 & 13.6235 & & 0.48 \\
\hline \multirow[t]{4}{*}{ 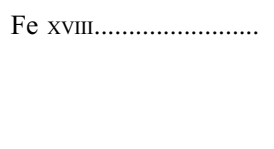 } & & $2 p^{4} 3 d^{2} D_{5 / 2} \rightarrow 2 p^{5}{ }^{2} P_{3 / 2}$ & 14.208 & $14.1776 \pm 0.0063$ & & $3.87 \pm 0.50$ \\
\hline & & $2 p^{4} 3 d^{2} D_{5 / 2} \rightarrow 2 p^{5}{ }^{2} P_{3 / 2}$ & 14.373 & 14.3423 & & 1.55 \\
\hline & & $2 p^{4} 3 s^{2} P_{3 / 2} \rightarrow 2 p^{5}{ }^{2} P_{3 / 2}$ & 16.004 & 15.9712 & & 2.32 \\
\hline & & $2 p^{4} 3 s^{2} P_{5 / 2} \rightarrow 2 p^{5}{ }^{2} P_{3 / 2}$ & 16.071 & 16.0381 & & $0.28 \pm 0.47$ \\
\hline \multirow[t]{9}{*}{ 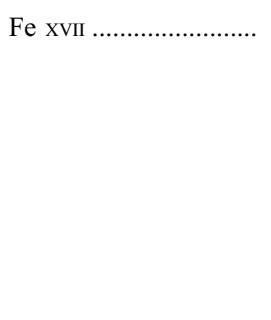 } & & $2 p^{5} 3 d^{1} P_{1} \rightarrow 2 p^{6}{ }^{1} S_{0}$ & 15.015 & $14.9828 \pm 0.0048$ & $83.1 \pm 9.3$ & $5.82 \pm 0.85$ \\
\hline & & $2 p^{5} 3 d^{3} D_{1} \rightarrow 2 p^{6}{ }^{1} S_{0}$ & 15.262 & 15.2340 & & $2.51 \pm 0.54$ \\
\hline & & $2 p^{5} 3 s{ }^{1} P_{1} \rightarrow 2 p^{6}{ }^{1} S_{0}$ & 16.778 & 16.7420 & & $0.94 \pm 0.51$ \\
\hline & & $2 p^{5} 3 s{ }^{3} P_{1} \rightarrow 2 p^{6}{ }^{1} S_{0}$ & 17.053 & 17.0168 & & $0.75 \pm 0.28$ \\
\hline & & $2 p^{5} 3 s{ }^{3} P_{2} \rightarrow 2 p^{6}{ }^{1} S_{0}$ & 17.096 & 17.0595 & & 0.75 \\
\hline & & $2 p^{5} 4 d^{3} D_{1} \rightarrow 2 p^{6}{ }^{1} S_{0}$ & 12.266 & 12.2435 & & $0.58 \pm 0.53$ \\
\hline & & $2 p^{5} 5 d^{3} D_{1} \rightarrow 2 p^{6}{ }^{1} S_{0}$ & 11.254 & 11.2329 & & $1.32 \pm 0.41$ \\
\hline & & $2 p^{5} 5 d^{1} P_{1} \rightarrow 2 p^{6}{ }^{1} S_{0}$ & 11.131 & 11.1102 & & $0.73 \pm 0.37$ \\
\hline & & $2 p^{5} 6 d^{3} D_{1} \rightarrow 2 p^{6}{ }^{1} S_{0}$ & 10.770 & 10.7498 & & $1.19 \pm 0.36$ \\
\hline
\end{tabular}



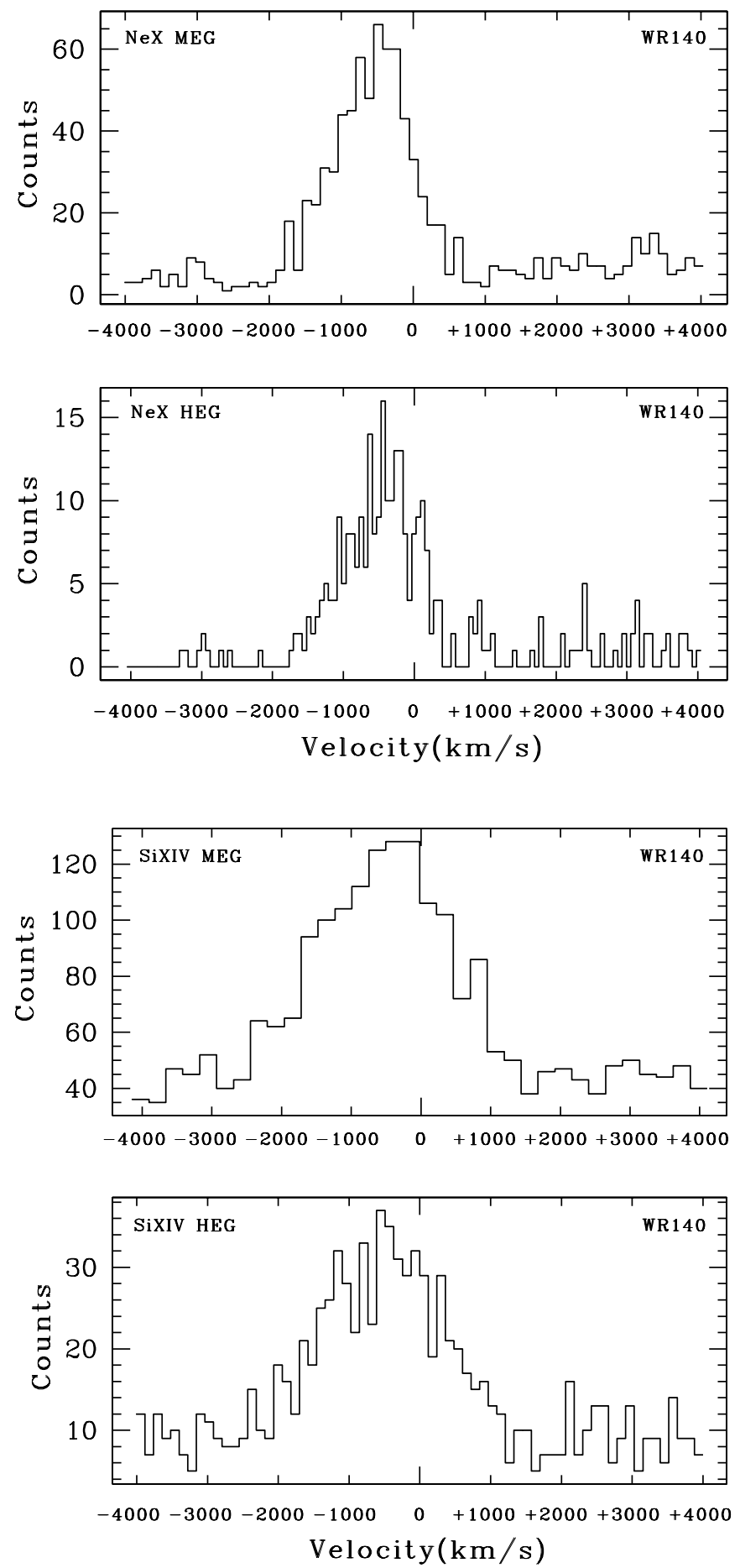

FIG. 6.-Combined positive and negative first-order MEG and HEG spectra of the Ne $\mathrm{x}$ and Si xIv Ly $\alpha$ lines in the bright WR 140 spectrum taken before periastron.

2001a), is more difficult to deal with in WR 140 , not least because of its relative weakness and the broadness of the lines. Nonetheless, all the ions between Fe XVII and Fe Xxv inclusive appeared in the pre-periastron spectrum. The strongest L-shell $(3 d-2 p)$ transitions were clearly visible of Fe XVII near $15.01 \AA$, Fe xVIII near 14.28 $\AA$, Fe xIx near 13.52 $\AA$, Fe xx near $12.85 \AA$, Fe XXI near $12.28 \AA$, and Fe XXII near $11.77 \AA$, along with the $(3 p-2 s)$ lines of Fe XxIII near $11.00 \AA$ and Fe xxIV near $10.60 \AA$. A few dozen other lines are also present, although often blended. In constructing multiple-line models, we have been guided by work on the spectra of Capella by Behar et al. (2001a) and of
TABLE 5

Velocity Structure of WR 140's Emission Lines before Periastron

\begin{tabular}{|c|c|c|c|}
\hline Ion & $\begin{array}{c}\Delta \lambda_{\mathrm{WR} 140} \\
(\mathrm{~m} \AA)\end{array}$ & $\begin{array}{c}v \\
\left(\mathrm{~km} \mathrm{~s}^{-1}\right)\end{array}$ & $\begin{array}{c}\sigma(v)_{\mathrm{WR} 140}{ }^{\mathrm{a}} \\
\left(\mathrm{km} \mathrm{s}^{-1}\right)\end{array}$ \\
\hline $\operatorname{Ar}$ XVIII . & $-5.0 \pm 5.1$ & $-402 \pm 410$ & $1448 \pm 1046$ \\
\hline Ar XVII ...... & $-7.1 \pm 6.7$ & $-539 \pm 509$ & $3065 \pm 1050$ \\
\hline 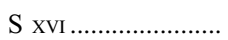 & $-9.0 \pm 2.5$ & $-571 \pm 159$ & $2541 \pm 394$ \\
\hline 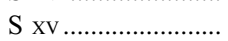 & $-13.3 \pm 1.8$ & $-791 \pm 107$ & $1623 \pm 251$ \\
\hline 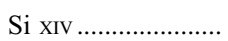 & $-13.6 \pm 0.8$ & $-660 \pm 39$ & $1663 \pm 92$ \\
\hline Si XIII ...................... & $-13.7 \pm 1.1$ & $-618 \pm 50$ & $1460 \pm 104$ \\
\hline $\mathrm{Al}$ XIII .................... & $-15.0 \pm 4.8$ & $-627 \pm 201$ & $1429 \pm 440$ \\
\hline 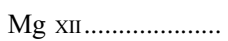 & $-18.4 \pm 1.4$ & $-655 \pm 50$ & $1770 \pm 103$ \\
\hline 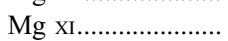 & $-17.4 \pm 1.9$ & $-569 \pm 62$ & $1245 \pm 128$ \\
\hline $\mathrm{Ne} \mathrm{x}$ & $-25.0 \pm 0.7$ & $-618 \pm 17$ & $1015 \pm 40$ \\
\hline 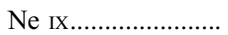 & $-28.3 \pm 3.2$ & $-631 \pm 71$ & $974 \pm 161$ \\
\hline O VIII.......................... & $-40.7 \pm 4.4$ & $-643 \pm 70$ & $849 \pm 147$ \\
\hline 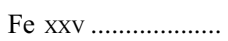 & -3.7 & -600 & $2876 \pm 552$ \\
\hline 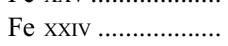 & $-13.5 \pm 2.4$ & $-381 \pm 68$ & $1210 \pm 150$ \\
\hline Fe XXI-Fe XXII ........ & $-13.9 \pm 2.8$ & $-355 \pm 72$ & $964 \pm 187$ \\
\hline Fe $x x$ & $-44.9 \pm 7.1$ & $-1050 \pm 166$ & $862 \pm 269$ \\
\hline Fe XIX & $-20.4 \pm 5.0$ & $-452 \pm 111$ & $862 \pm 269$ \\
\hline 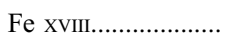 & $-30.4 \pm 6.3$ & $-641 \pm 133$ & $1663 \pm 186$ \\
\hline 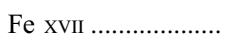 & $-32.2 \pm 4.8$ & $-643 \pm 96$ & $1663 \pm 186$ \\
\hline
\end{tabular}

${ }^{\text {a }}$ Velocity FWHM.

$\theta^{1}$ Orionis $\mathrm{C}$ by Schulz et al. (2003) and especially by the Brown et al. (2002) EBIT laboratory measurements of Fe XVIII-XXIV between 10.6 and $18 \AA$, whose line ratios we have adopted where blending caused problems.

\subsection{The Faint Post-Periastron Observation of 2001 May 8}

WR 140 was observed again in early 2001 May when events conspired to reduce the number of detected counts by nearly an order of magnitude as shown in Table 2. The system was further from periastron making it intrinsically fainter, the eclipse was still underway, and the exposure time was shorter. The considerable extra absorption is obvious, limiting the useful spectrum to wavelengths shorter than about $10 \AA$, due to the intervention of cool WC material between the observer and the X-ray-bright interaction region between the stars. The HEG and MEG spectra are shown in Figure 9. Only the lines of $\mathrm{Si}, \mathrm{S}$, and Fe xxv obviously survived in the absorbed post-periastron spectrum. Although the lines were relatively weakly detected, their velocity structure was significantly different from the earlier observation just over 4 months before: they were wider and all shifted marginally to the red rather than substantially to the blue. The data are not good enough to derive a full set of individual ion velocities so we estimated a single velocity shift and width for all of the Si and S H-like and He-like lines together along with a suitably wide stretch of polynomial continuum. At even shorter wavelengths, where the effects of absorption were less important, the Fe xxv triplet was reasonably well detected. With the intercombination line intensity fixed at zero from the pre-periastron measurement, its total flux was fainter by a factor of $1.5 \pm 0.3$, consistent with the ratio of the binary separations. The line fluxes and widths are shown in Table 7 and the corresponding velocities in Table 8. The strongest Si XIII line is shown in Figure 10 and has a suggestion of double structure.

\section{PHYSICAL CONDITIONS IN WR 140}

\subsection{The Overall X-Ray Spectrum and Continuum Temperature}

Although there were many prominent lines in WR 140's preperiastron spectrum, the 70 or so lines in Tables 3 and 4 account 


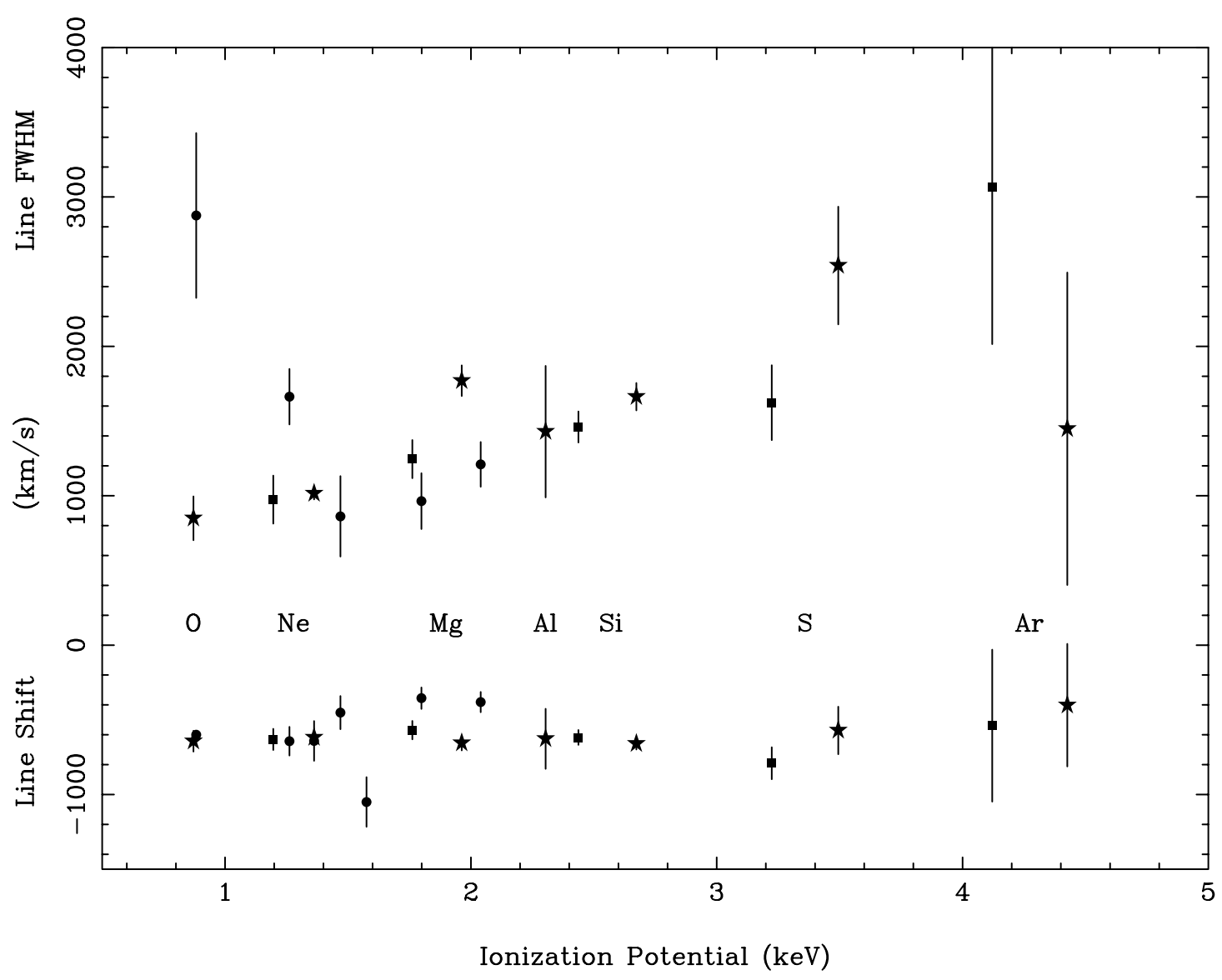

FIG. 7.- - Line shifts and FWHM of the emission lines in the bright WR 140 spectrum taken before periastron plotted against ionization potential. The H-like lines of $\mathrm{O}, \mathrm{Ne}, \mathrm{Mg}, \mathrm{Al}, \mathrm{Si}, \mathrm{S}$, and $\mathrm{Ar}$ are plotted by stars and the He-like lines by squares. Problems of weakness, blending, and confusion make the lines of the various stages of Fe less reliable, and they are indicated by circles.

for less than $20 \%$ of the overall flux because of the hot, strong continuum. In contrast to the lines, the continuum arises, of course, essentially from the free electrons in the plasma. The relative energetics of ions and electrons is one of the important aspects to be determined of any shock-heated plasma. Although we relied above on empirical models of the local continuum to study the velocity profiles of individual lines, we were also able to model the whole spectrum with a combination of lines and a broadband continuum. In place of the piecewise empirical polynomial background we first used a bremsstrahlung continuum with the complete set of individual lines, whose parameters were allowed to vary from their locally determined values, resulting in what we expect are reliable estimates of the continuum temperature, absorbing column density, and luminosity as reported in Tables 9 and 10. The fit to the whole spectrum is good and implies an absorbing column density not far from that expected from interstellar reddening, as discussed below, and a continuum or electron temperature of nearly $5 \mathrm{keV}$. This temperature may be compared with the theoretical value, $T_{s}=1.96 \bar{m} v_{8}^{2} \mathrm{keV}$, expected after thermalization in the strong-shock ideal-gas limit for material of mean particle mass, $\bar{m}$, in units of the proton mass, flowing into a stationary shock at a typical wind terminal velocity $v_{\infty}=1000 v_{8} \mathrm{~km} \mathrm{~s}^{-1}$. For the WC wind in WR 140, $T_{s} \sim 25 \mathrm{keV}$, considerably higher than observed.

It is unlikely that the intrinsic emission from the wind of either star made any significant contribution to the observations. The preperiastron luminosity was about an order of magnitude higher than the most luminous O star, itself a binary, in the ROSAT All-Sky Survey (Berghöfer et al. 1996) and about 2 orders of magnitude brighter than more typical single-star luminosities, and single WC stars are notably faint X-ray sources (Oskinova et al. 2003). Coupled with the unusually high temperature and the systematic participation of all the detected lines in the coherent velocity flows implied by Figure 7, both stars can confidently be excluded from further discussion.

\subsection{Chemical Composition}

Ignoring for a moment the inhomogeneity of the plasma demonstrated by the variety of observed line widths, we tried to assess how well the overall properties of the ensemble of lines and continuum could be reproduced by the optically thin plasma models offered by XSPEC and CIAO. Although there are two winds colliding in WR 140, Pittard \& Stevens (2002 and references therein) have argued that the emission is dominated by the much more powerful wind of the WC star, and we continued on this basis. The grating spectrum then offers the chance to use variable-abundance plasma models to measure the chemical composition of the X-ray-bright Wolf-Rayet material. The abundances in the plasma models are given with respect to hydrogen, which is absent in WC stars. We treated this by fixing a very high helium abundance that then served as the reference for other elements. The observed spectrum constrains directly elements between $\mathrm{O}$ and $\mathrm{Fe}$ with lines, detected or not, that fall in the wavelength range covered by the observations. The continuum, on the other hand, arises from free electrons stripped largely from the otherwise unobservable but more abundant lighter elements $\mathrm{He}$ and $\mathrm{C}$. In general terms, within the confines of the physics that underlies the plasma models, in the presence of a strong 

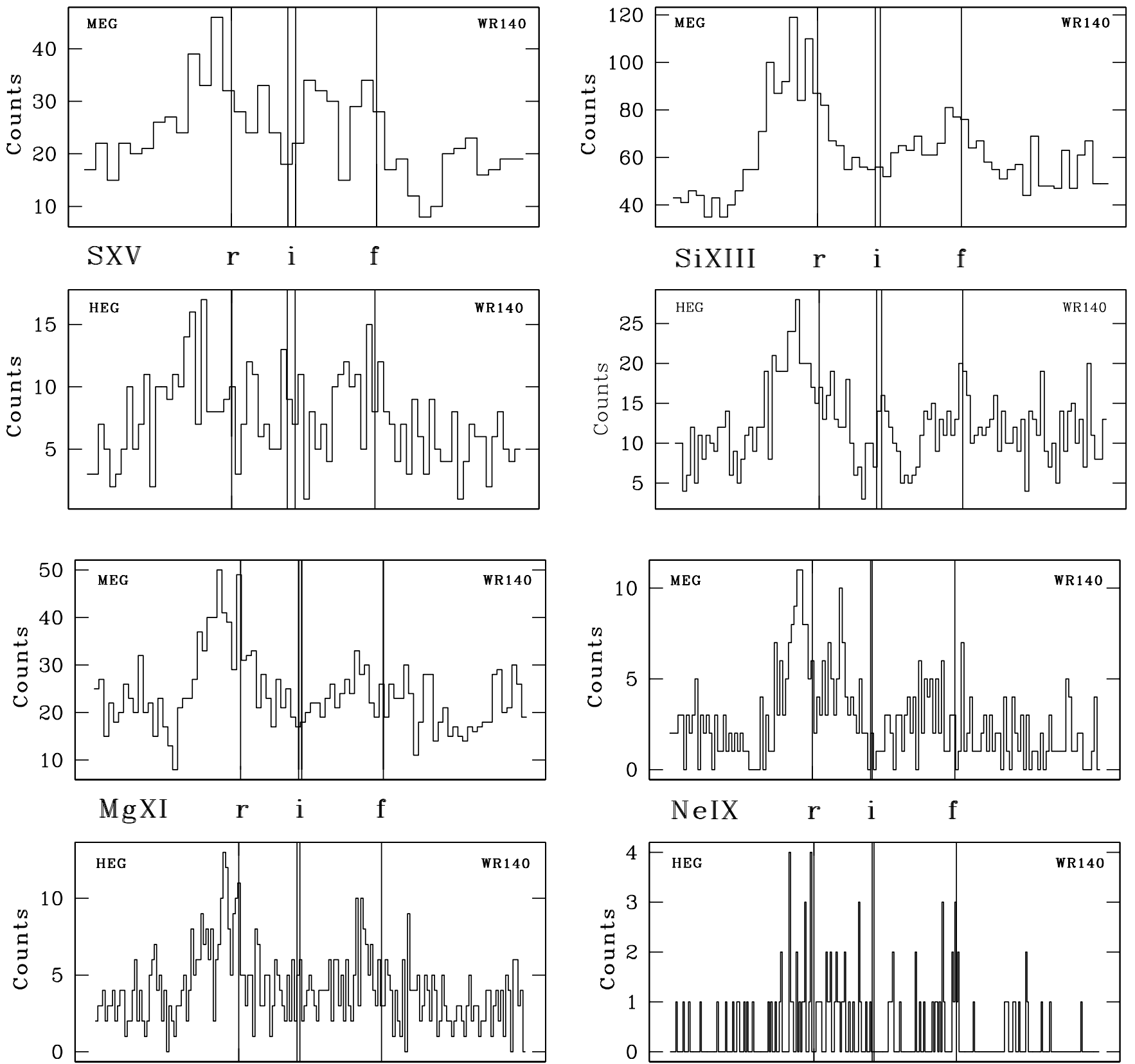

FIG. 8.-He-like triplets of S Xv, Si XIII, Mg XI, and Ne IX at full resolution in the bright spectrum taken of WR 140 before periastron showing the general weakness of the intercombination lines. Most of the emission between Ne IX $r$ and $f$ is due to the strongest lines of Fe XIX, namely, $\lambda 13.518$ and its nearby companions.

TABLE 6

He-like Triplet Measurements of WR 140 on 2000 December 29

\begin{tabular}{|c|c|c|c|}
\hline Line & $G=(i+f) / r$ & $R=f / i$ & $R 0$ \\
\hline $\mathrm{S} x \mathrm{xV} \ldots \ldots \ldots \ldots \ldots$ & $1.00_{-0.24}^{+0.30}$ & $3.0_{-1.2}^{+4.3}$ & 2.1 \\
\hline Si XIII .................. & $0.74_{-0.08}^{+0.10}$ & $2.3_{-0.5}^{+0.8}$ & 2.8 \\
\hline 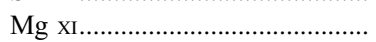 & $0.55_{-0.11}^{+0.10}$ & $5.0_{-2.3}^{+15.3}$ & 3.1 \\
\hline Ne IX................................... & $0.55_{-0.14}^{+0.12}$ & $>4.0$ & 3.4 \\
\hline
\end{tabular}

Note. $-R 0$ is the upper limit determined by atomic physics of the relative intensity of the forbidden and intercombination lines at low densities in the absence of UV radiation. continuum an elemental abundance is determined almost directly from a line's equivalent width. Observationally, it is not possible to distinguish between $\mathrm{He}$ and $\mathrm{C}$ so we used two values of the ratio $\mathrm{C} / \mathrm{He}=0.1$ and $\mathrm{C} / \mathrm{He}=0.4$ that cover the range of values in both the observational work of Eenens \& Williams (1992), who included WR 140 itself, and Dessart et al. (2000) and the more general considerations of van der Hucht et al. (1986). Tables 9 and 10 report the individual element abundances relative to He among other relevant parameters of the isothermal models considered. Results derived using XSPEC and Sherpa were identical. The likelihood $C$-statistic given in the tables allows direct comparison of the models and also has a property similar to $\chi^{2}$ in that its reduced value is close to unity when the fit is good. The lines+continuum model had about 200 free parameters, nearly all of which were the same individual lines or groups of lines that appear in Tables 3 and 4, which were all fitted simultaneously in this case. 


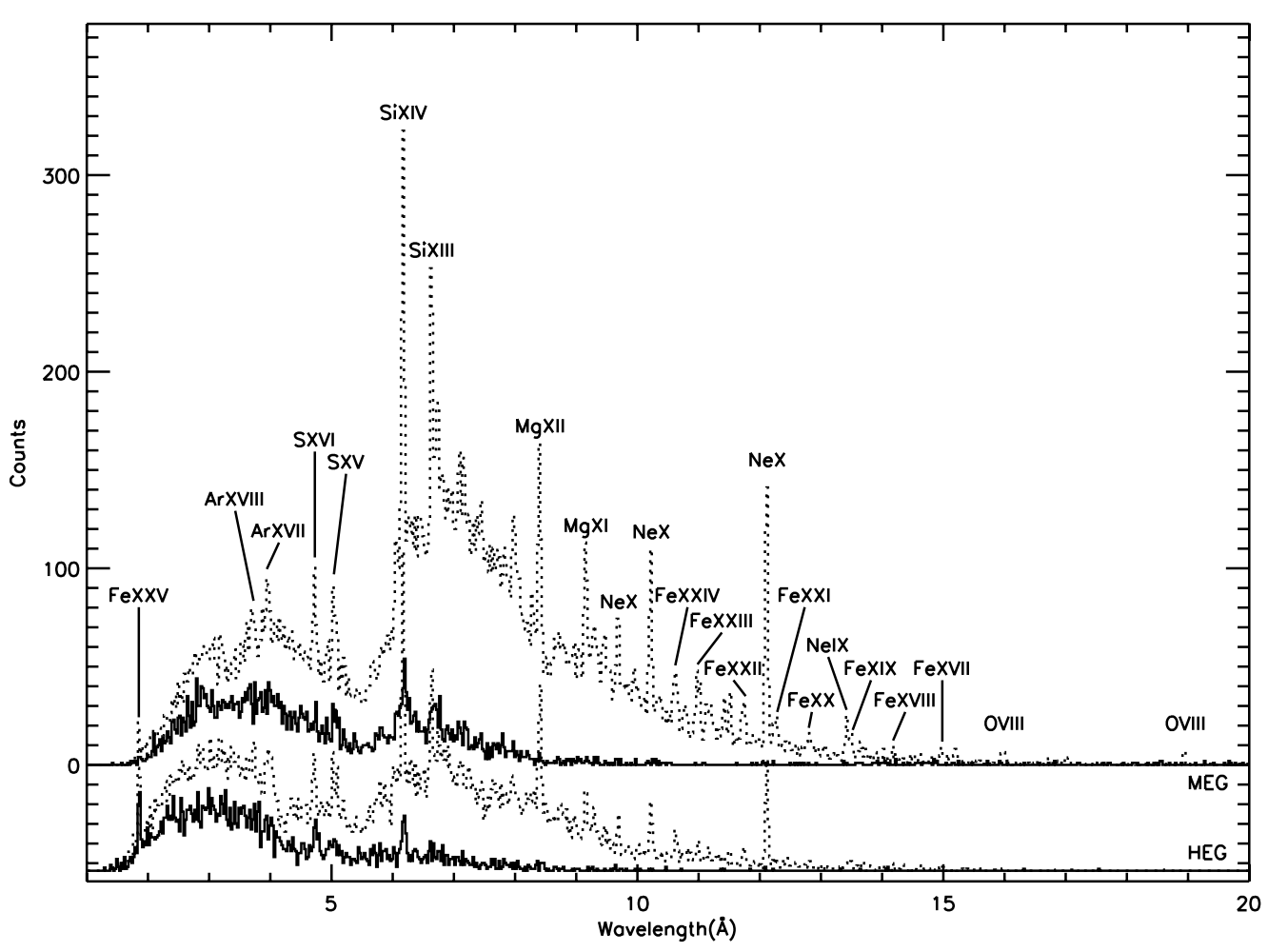

FIG. 9.-WR 140's combined first-order Chandra HEG and MEG spectra during the post-periastron observation of 2001 May 8 showing mainly the extra absorption by the intervening wind of the WC star. The dotted lines show the pre-periastron spectra of Fig. 4 scaled by the relative exposure times.

Given the simplicity of the isothermal models, they worked very well and were only really deficient in their inability to account for any of the prominent He-like lines, in particular, or the weak lines from cooler Fe ions. The broad lines, which might have been expected to cause trouble, were reasonably well but accidently reproduced by spurious broadening in the model. Highresolution data are a stringent test of all aspects of both the physics and numerical implementation of the available spectral models. Individual line models represent narrow lines well. The multiple-line plasma models were generally developed before high-resolution data for the purposes of studying the overall balance between different ions and not the individual shapes of lines. Given the new challenges that they now face, the success of these models is remarkable.

Because the models fail to account for the He-like triplets, we considered adjusting the observed abundances by a ratio $(1+$ $f_{\mathrm{He}} / f_{\mathrm{H}}$ ) calculated from the fluxes of the $\mathrm{H}$-like and He-like lines but decided to await a more complete treatment of the ionization balance as sketched out below. The ratio of about 2.4 between the abundances for $\mathrm{C} / \mathrm{He}=0.1$ and 0.4 is largely due to the $Z^{2}$ dependence of the bremsstrahlung emissivity that thus scales as $\left[2^{2}+(\mathrm{C} / \mathrm{He}) 6^{2}\right]$ for the mixture of $\mathrm{He}(Z=2)$ and $\mathrm{C}(Z=6)$ responsible for most of the continuum. It is an easy task to scale the abundances to any other value of $\mathrm{C} / \mathrm{He}$.

Abundances in WC stars are difficult to measure at longer wavelengths, but these X-ray-determined values should provide an important reference point. They compare well, for example, with the Dessart et al. (2000) IR-derived values: WR 140's value of $\mathrm{Ne} / \mathrm{S}=43 \pm 4$, which is independent of the unknown relative abundances of $\mathrm{He}$ and $\mathrm{C}$, agrees well with their value of 50 for $\gamma$ Velorum, which they pointed out is 8 times greater than the cosmic value due to enhancement of $\mathrm{Ne}$. It does indeed seem likely that $\mathrm{Ne}$ is enhanced in WR 140, given the notable strength of the $\mathrm{Ne} \operatorname{Ly} \alpha$ and $\operatorname{Ly} \beta$ lines in comparison with the Fe lines

TABLE 7

WR 140's Emission Lines after Periastron

\begin{tabular}{|c|c|c|c|c|c|}
\hline Ion & Line & $\begin{array}{l}\lambda_{v=0} \\
(\AA)\end{array}$ & $\begin{array}{l}\lambda_{\mathrm{WR}} 140 \\
(\AA)\end{array}$ & $\begin{array}{c}\text { FWHM } \\
(\mathrm{m} \AA)\end{array}$ & $\begin{array}{c}\text { Flux } \\
\left(10^{-5} \mathrm{~cm}^{-2} \mathrm{~s}^{-1}\right)\end{array}$ \\
\hline \multirow[t]{3}{*}{ Fe xxv ........................ } & $r$ & 1.8500 & $1.8540 \pm 0.0016$ & $10.4_{-5.4}^{+4.8}$ & $9.95_{-1.61}^{+1.74}$ \\
\hline & $i$ & 1.8595 & 1.8635 & 10.4 & 0.00 \\
\hline & $f$ & 1.8682 & 1.8723 & 10.5 & $1.80_{-1.07}^{+1.21}$ \\
\hline $\mathrm{S}$ XVI & $\operatorname{Ly} \alpha$ & 4.7274 & 4.7299 & 47.3 & $2.55 \pm 0.54$ \\
\hline \multirow[t]{3}{*}{$\mathrm{S}$ xV } & $r$ & 5.0387 & 5.0413 & 50.4 & $3.75 \pm 0.86$ \\
\hline & $i$ & 5.0665 & 5.0691 & 50.7 & $0.00 \pm 0.96$ \\
\hline & $f$ & 5.1015 & 5.1041 & 51.0 & $1.83 \pm 0.77$ \\
\hline Si XIV ............ & $\operatorname{Ly} \alpha$ & 6.1804 & $6.1836 \pm 0.0035$ & $61.8_{-6.3}^{+7.3}$ & $2.08 \pm 0.28$ \\
\hline \multirow[t]{3}{*}{ 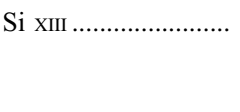 } & $r$ & 6.6480 & 6.6515 & 66.5 & $1.19 \pm 0.32$ \\
\hline & $i$ & 6.6883 & 6.6918 & 66.9 & $0.54 \pm 0.33$ \\
\hline & $f$ & 6.7404 & 6.7439 & 67.4 & $0.81 \pm 0.27$ \\
\hline
\end{tabular}


TABLE 8

Velocity Structure of WR 140's Emission Lines after Periastron

\begin{tabular}{|c|c|c|c|}
\hline Ion & $\begin{array}{c}\Delta \lambda_{\mathrm{WR}} 140 \\
(\mathrm{~m} \AA)\end{array}$ & $\begin{array}{c}v \\
\left(\mathrm{~km} \mathrm{~s}^{-1}\right)\end{array}$ & $\begin{array}{c}\sigma(v)_{\mathrm{WR} 140}{ }^{\mathrm{a}} \\
\left(\mathrm{km} \mathrm{s}^{-1}\right)\end{array}$ \\
\hline Fe xxv ....................... & $+4.0 \pm 1.6$ & $+648 \pm 259$ & $1676_{-876}^{+778}$ \\
\hline 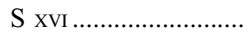 & +2.4 & $\ldots$ & $\ldots$ \\
\hline 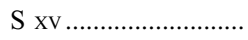 & +2.6 & $\ldots$ & $\ldots$ \\
\hline 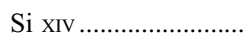 & $+3.2 \pm 3.5$ & $+159 \pm 167$ & $3000_{-305}^{+355}$ \\
\hline 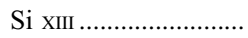 & +3.4 & $\ldots$ & $\ldots$ \\
\hline
\end{tabular}

${ }^{a}$ Velocity FWHM.

nearby; in Capella and $\theta^{1}$ Orionis $\mathrm{C}$ the $\mathrm{Fe}$ lines predominate. Gräfener et al. (1998) and Hillier \& Miller (1999) have discussed WC O abundances in the Galaxy and the LMC derived from spectral models of optical and UV data and show plainly the complications involved. For Hillier and Miller's best-fit $\mathrm{C} / \mathrm{He}=$ 0.4 for the WC5 star WR 111, our O/He from Table 9 of $0.072 \pm$ 0.001 is also consistent with their preferred although uncertain value of 0.1 . This apparent success implies that these interim $\mathrm{X}$-ray abundance measurements of the other elements are also worth taking seriously. We have used the best-fit WC abundances for $\mathrm{C} / \mathrm{He}=0.4$ in calculations below involving physical conditions in the Wolf-Rayet wind to define mean values of the atomic weight $\mu=6.89$, ionic charge $Z=3.44$, and particle mass of fully ionized ions and electrons $\bar{m}=1.55$ in units of the proton mass.

\subsection{Absorption by the Wind of the O Star}

The best-fit pre-periastron absorbing column densities of Table 9 depend on the spectral model. Of the three, the highest value from the best-fit, least-constrained lines+wabs*bremss model is probably the most reliable for comparison with that expected from the star's interstellar reddening given in Table 1: the best-fit value is about $30 \%$ higher. The X-ray source was being observed through the $\mathrm{O}$ star wind, and the corresponding absorption must be present at some level depending on the wind parameters and the geometry of the line of sight. An estimate can be made using the stellar parameters in Table 1 and the binary system separation at the time of the observation in Table 2 . Starting from the mass continuity equation for a spherically symmetric wind

$$
\dot{M}=4 \pi r^{2} \mu m_{p} n_{i}(r) v(r),
$$

where $\mu$ is the mean atomic number, $m_{p}$ is the proton mass, and $n_{i}(r)$ is the ion density and assuming a $\beta$ velocity law $v(r)=$ $v_{\infty}\left(1-R_{*} / r\right)^{\beta}$, the expected column density of all ions $N_{i}=$ $\int n_{i}(r) d l$ between a point distance $R$ from the surface of the star along the line of sight may be written as

$$
\begin{aligned}
N_{i}(R, \phi, i) \sim & 4.31 \times 10^{23} \dot{M}_{-6} \mu^{-1} v_{8}^{-1}\left(R_{*} / R_{\odot}\right)^{-1} \\
& \times H\left(R / R_{*}, \beta\right)(\gamma / \sin \gamma) \mathrm{cm}^{-2}
\end{aligned}
$$

where $\cos \gamma=\cos \phi \sin i$ and

$$
H\left(R / R_{*}, \beta\right)=\int_{R / R_{*}}^{\infty} x^{-2}(1-1 / x)^{-\beta} d x,
$$

with $\dot{M}=\dot{M}_{-6} \times 10^{-6} M_{\odot} \mathrm{yr}^{-1}$ and $v_{\infty}=1000 v_{8} \mathrm{~km} \mathrm{~s}^{-1}$. The angle $\phi$ is the azimuthal angle between the line joining the stars
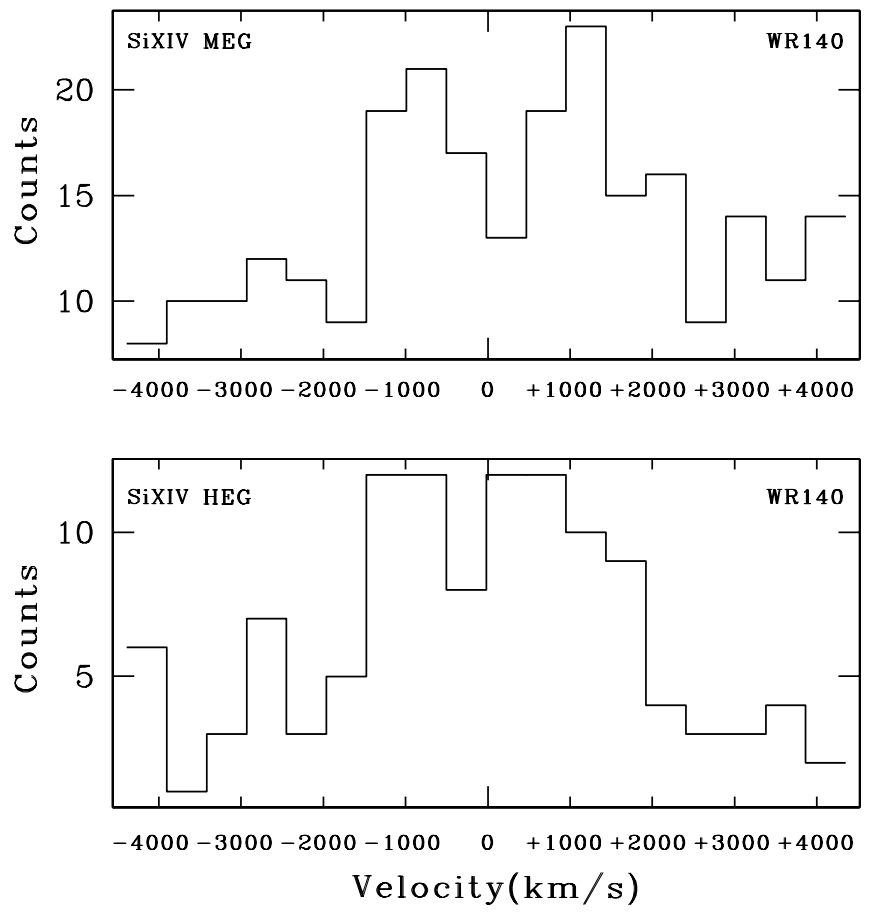

FIG. 10.-Combined positive and negative first-order MEG and HEG spectra of the Si XIV Ly $\alpha$ lines in the faint WR 140 spectrum taken after periastron.

and the line of sight, and $i$ is the inclination. The increase in $N_{i}$ for lines of sight that pass close to the $\mathrm{O}$ star is described approximately by the geometrical factor $\gamma / \sin \gamma$, which increases from unity for the radial line of sight away from the star defined by $(\gamma, i)=(0,0)$ by more than an order of magnitude for those that pass within a few degrees of the $\mathrm{O}$ star. For $R / R_{*} \gg 1, H \sim$ $1 /\left(R / R_{*}\right)$.

For the O star in WR $140 \phi=(\nu-\omega)$ for the orbital true anomaly $\nu$ in Table 2. Hydrodynamic models (e.g., Stevens et al. 1992) confirm that the stagnation point is fixed by momentum balance between the opposing winds and for WR 140 put it at about $\frac{1}{5}$ of the binary separation from the $\mathrm{O}$ star, so that $R \approx$ $(D / 5)$ or about $12.4 R_{\mathrm{O}} / \sin i$ during the pre-periastron observation when $\phi=220^{\circ}$. . Inserting other appropriate system parameters from Table 1 and converting $N_{i}$ to the equivalent hydrogen column density gives

$$
N_{\mathrm{H}}^{\mathrm{O}} \sim 3.0 \times 10^{21} \mathrm{~cm}^{-2},
$$

which is about half of that expected from interstellar absorption. Both estimates are subject to uncertainties that are quite hard to quantify. While the error on the expected interstellar value quoted in Table 1 relates to the spread in the ensemble averages compiled by Vuong et al. (2003), it is clear that the column densities of individual objects show a greater than statistical spread presumably due, at least in part, to variations in the gas-to-dust ratio. Similarly, the expected O star circumstellar value depends most obviously on the mass-loss rate but is also sensitive at the level of a few tens of percent to uncertainties in the system geometry and to any differential absorption due to the extended nature of the X-ray source that we have ignored. With these caveats, the expected total of circumstellar and interstellar absorption is then

$$
N_{\mathrm{H}} \sim(8.9 \pm 1.0) \times 10^{21} \mathrm{~cm}^{-2},
$$


TABLE 9

Broadband Parameters of 2000 December 29 Pre-Periastron WR 140 X-Ray Spectra

\begin{tabular}{|c|c|c|c|}
\hline \multirow[b]{2}{*}{ PARAMETER } & \multirow[b]{2}{*}{ lines+wabs*bremss } & \multicolumn{2}{|c|}{ wabs*vmekal } \\
\hline & & $\mathrm{C} / \mathrm{He}=0.1$ & $\mathrm{C} / \mathrm{He}=0.4$ \\
\hline 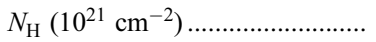 & $7.78 \pm 0.05$ & $6.60 \pm 0.04$ & $6.52 \pm 0.04$ \\
\hline 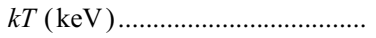 & $4.88 \pm 0.05$ & $3.95 \pm 0.05$ & $3.87 \pm 0.05$ \\
\hline Не & $\ldots$ & $1 .^{\mathrm{a}}$ & $1 .^{\mathrm{a}}$ \\
\hline (n) & $\ldots$ & $0.1^{\mathrm{a}}$ & $0.4^{\mathrm{a}}$ \\
\hline ................. & $\ldots$ & $(3.19 \pm 0.05) \times 10^{-2}$ & $(7.18 \pm 0.12) \times 10^{-2}$ \\
\hline $\mathrm{Ne}$ & $\ldots$ & $(0.42 \pm 0.01) \times 10^{-2}$ & $(1.03 \pm 0.03) \times 10^{-2}$ \\
\hline 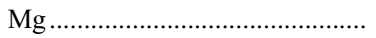 & $\ldots$ & $(2.63 \pm 0.15) \times 10^{-4}$ & $(6.54 \pm 0.38) \times 10^{-4}$ \\
\hline $\mathrm{Al} . \ldots \ldots \ldots \ldots$ & $\ldots$ & $(0.81 \pm 0.58) \times 10^{-5}$ & $(2.04 \pm 1.44) \times 10^{-5}$ \\
\hline $\mathrm{Si}$ & $\ldots$ & $(2.34 \pm 0.09) \times 10^{-4}$ & $(5.85 \pm 0.23) \times 10^{-4}$ \\
\hline 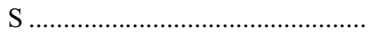 & $\ldots$ & $(0.96 \pm 0.08) \times 10^{-4}$ & $(2.41 \pm 0.19) \times 10^{-4}$ \\
\hline 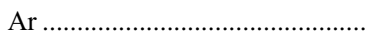 & $\ldots$ & $(2.15 \pm 0.37) \times 10^{-5}$ & $(5.37 \pm 0.92) \times 10^{-5}$ \\
\hline $\mathrm{Fe}$ & $\ldots$ & $(0.68 \pm 0.45) \times 10^{-4}$ & $(1.69 \pm 0.11) \times 10^{-4}$ \\
\hline$z$ & $\ldots$ & -0.0016 & -0.0016 \\
\hline 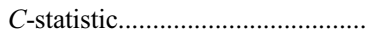 & 17831.9 & 20042.9 & 20021.6 \\
\hline Number of bins ........................... & 19204 & 19204 & 19204 \\
\hline$L_{X}{ }^{\mathrm{b}}\left(10^{34} \operatorname{ergs~s}^{-1}\right) \ldots \ldots \ldots \ldots \ldots \ldots \ldots \ldots \ldots \ldots \ldots \ldots$ & 1.25 & 1.21 & 1.21 \\
\hline$L_{\mathrm{X}}, N_{\mathrm{H}}=0^{\mathrm{c}}\left(10^{34} \mathrm{ergs} \mathrm{s}^{-1}\right) \ldots \ldots$ & 2.04 & 1.49 & 1.48 \\
\hline
\end{tabular}

${ }^{\mathrm{a}}$ Fixed value. Quoted errors elsewhere are $1 \sigma$.

b $(0.5-10 \mathrm{keV})$ at $1.1 \mathrm{kpc}$. The luminosity measurement errors are negligible.

c Corrected for interstellar and circumstellar absorption.

which is reasonably consistent with the best-fit values reported in Table 9. Measurements made in the past with lower resolution instruments observing through the $\mathrm{O}$ star wind between apastron and periastron (Pollock et al. 2002) have shown little evidence of the low-energy spectral variations that might have been expected for different lines of sight through a significantly absorbing $\mathrm{O}$ star wind. In the light of the Chandra results, we intend to reassess the currently available data in the forthcoming $R X T E$ paper, although the matter will probably only be settled with new high-resolution measurements.

\subsection{Absorption by the Wind of the WC Star}

When the unshocked Wolf-Rayet wind intervenes in the line of sight, absorption is strong. We had anticipated the chance of being able to use the absorption spectrum to make an independent assessment of the elemental abundances of WC-type material by comparing models of different abundance schemes. However, the interstellar absorption has already removed any possibility of observing an O edge near $23.3 \AA$, and with the extra suffered through the Wolf-Rayet wind, the edges of Ne near

TABLE 10

Broadband Parameters of 2001 May 8 Post-Periastron WR 140 X-RAY SPECTRA

\begin{tabular}{|c|c|}
\hline Parameter & $\begin{array}{c}\text { wabs*vmekal } \\
\mathrm{C} / \mathrm{He}=0.4\end{array}$ \\
\hline$N_{\mathrm{He}}\left(10^{20} \mathrm{~cm}^{-2}\right) \ldots \ldots \ldots \ldots \ldots$ & $2.83 \pm 0.08$ \\
\hline 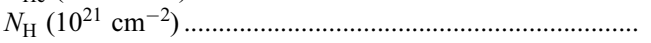 & $6.52^{\mathrm{a}}$ \\
\hline$z$ & $0 .^{\mathrm{a}}$ \\
\hline$C$-statistic.................... & 9732.9 \\
\hline 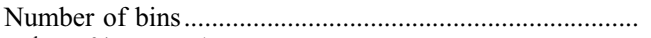 & 10796 \\
\hline$L_{\mathrm{X}}^{\mathrm{b}}\left(10^{34} \operatorname{ergs~s}^{-1}\right) \ldots$. & 0.51 \\
\hline 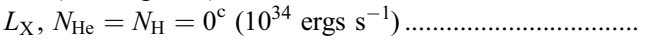 & 1.03 \\
\hline
\end{tabular}

${ }^{\text {a }}$ Fixed value. Quoted errors elsewhere are $1 \sigma$.

b $(0.5-10 \mathrm{keV})$ at $1.1 \mathrm{kpc}$. The luminosity measurement errors are negligible.

${ }^{c}$ Corrected for interstellar and circumstellar absorption.
14.3 $\AA$, in particular, and Mg near $9.5 \AA$ were also obliterated, leaving it impossible to distinguish between the elements responsible for the bulk of circumstellar absorption. The edges of Si near $6.7 \AA$ and $\mathrm{S}$ near $5.0 \AA$ lie in regions complicated by both colliding-wind emission lines and instrumental features. In these circumstances, we made the composition of an extra component of cold circumstellar X-ray absorbing material the same as the hot emitting material with $\mathrm{C} / \mathrm{He}=0.4$ in Table 9 keeping all the other emission parameters the same except $z=0$ and a free normalization. The best-fit He column density is

$$
N_{\mathrm{He}}^{\mathrm{WC}}=(2.83 \pm 0.08) \times 10^{20} \mathrm{~cm}^{-2} .
$$

This is roughly equivalent to about $3.05 \times 10^{22} \mathrm{~cm}^{-2}$ of solar composition material or $2.01 \times 10^{20} \mathrm{~cm}^{-2}$ of pure carbon. The measurement does give a direct estimate of the WC mass-loss rate. Using the appropriate geometry and system parameters including the mass-loss rate and converting $N_{i}$ to the equivalent helium column density gives an expected value of

$$
N_{\mathrm{He}}^{\mathrm{WC}} \sim 10.7 \times 10^{20} \mathrm{~cm}^{-2},
$$

which is roughly a factor of 4 greater than observed. Although this could be most obviously interpreted as showing by how much the mass-loss rate could be reduced, we prefer to offer it as a high-resolution reference point to a discussion deferred to our forthcoming $R X T E$ paper that covers the entire superior conjunction of the Wolf-Rayet star shown in Figure 1 whose minimum, incidentally, coincides with the superior conjunction of the WC star in the Marchenko et al. (2003) orbit.

\subsection{The Ionization State of the Shocked Gas}

The simultaneous presence of H-like and He-like lines is a property of recently observed X-ray spectra of other hot-star systems thought to contain colliding winds. While Skinner et al. (2001) appealed generally to a multitemperature plasma in $\gamma$ Velorum, Corcoran et al. (2001) suggested that the two sets of lines in $\eta$ Carinae's spectrum are identified with the fast and slow 
winds of companion stars. As WR 140's WC7 and O4-5 stars both have winds with terminal velocities near $3000 \mathrm{~km} \mathrm{~s}^{-1}$, the latter idea does not obviously apply. The similarity of the velocity structure of WR 140's H-like and He-like lines suggests a common, although not completely common, origin. The trend in Table 5 and Figure 7 is for low-ionization lines to have consistently lower velocity widths than the higher ionization lines, both between $\mathrm{H}$-like and He-like ions and between heavier and lighter elements. Any macroscopic shock model, essentially by definition, is bound to feature low-velocity material near the stagnation point, where the velocity of an infinitesimally small amount of gas is zero, to values approaching the undisturbed wind velocities far away as gas flows out of the system. We can think of no reason to question this principle whatever the physics involved. Figure 7 is then direct evidence for evolution of the ionization of the outflowing gas. The relatively narrow widths of $\mathrm{H}$-like $\mathrm{O}$ VIII and $\mathrm{Ne} x$ show that they are more confined near the stagnation point than the production of Si XIV, for example, which takes place in a larger volume extending to higher velocities.

Similarly, the lower velocity widths of He-like triplets in comparison with their $\mathrm{H}$-like counterparts shows that their production is more centrally confined and that they are probably being produced by a plasma out of equilibrium and ionizing rather than cooling in more or less the collisional equilibrium that underlies the plasma codes usually used to predict line ratios and other spectral diagnostics. If the $R=f / i$ ratios are higher than the low-density limit $R 0$, and this is certainly the case in $\eta$ Carinae, then the strength of the forbidden lines could have been boosted by innershell ionization of Li-like ions as discussed theoretically by Mewe \& Schrijver (1978) and observed in the laboratory by Decaux et al. (1997), for example, and in the X-ray spectrum of the SNR N132D by Behar et al. (2001b). In WR 140's case, these Li-like ions would appear soon after the shock transition as a temporary stage during the establishment of thermal and ionization equilibrium in the outflowing gas. Within the framework of stagnation point flow, the lower velocity widths of the He-like triplets exclude their production farther out. Calculations of the expected level of inner-shell ionization would need to specify the ionizing source, which could conceivably be either thermal or nonthermal.

\subsection{Flow Geometry of the Colliding Winds}

The hydrodynamic models of Stevens et al. (1992), in particular their Figure 18, give the basis for understanding the observations of WR 140 before and after the 2001 periastron passage. Henley et al. (2003) have recently calculated the line profiles to be expected from such typical models of colliding winds, incorporating normal assumptions concerning the energy budget at the shock transition and the equilibrium state of the postshock gas that there has been no reason to question until now. They predict a variety of line shapes depending on the viewing geometry and binary parameters, including the types of singlepeaked and double-peaked profiles observed in WR 140 before and after periastron in Figures 6 and 10, respectively. The onset of the eclipse in Figure 1 only about 10 days after the first Chandra spectrum was taken shows that in late December of 2000 the line of sight was directly along the interaction region, also known as the shock cone. Hot gas moving out along the cone accounts for the range of mainly blueshifted material seen in that spectrum. This should include the highest velocity X-ray gas ever observed from WR 140. A few months later in 2001 May, superior conjunction of the Wolf-Rayet star had already passed, hidden from X-ray view by extreme absorption, and the cone had swung round.

The question is not so much why are the lines so broad, but rather why are some so narrow? Line broadening should arise principally from the postshock Doppler velocity dispersion, with contributions from both acceleration away from the stagnation point and the changing line of sight to different parts of the axisymmetric azimuthal flow. The width of the narrowest line $\mathrm{O}$ VIII is probably mostly due to its azimuthal distribution, although its thermal width is not negligible. The Maxwellian dispersion $v_{\text {thermal }}=$ $\left(8 k T / \pi m_{i}\right)^{1 / 2}$ implies FWHM thermal $\sim 820\left(T_{\mathrm{keV}} / A_{i}\right)^{1 / 2} \mathrm{~km} \mathrm{~s}^{-1}$ for an ion of atomic weight $A_{i}$ in a plasma of temperature $T_{\mathrm{keV}}$. For O at $5 \mathrm{keV}$, this is about $450 \mathrm{~km} \mathrm{~s}^{-1}$.

Following reasoning similar to Lührs (1997) and considering an ideal narrow ring of $\mathrm{O}$ VIII emission from material moving at velocity $v_{0}$ at an angle $\theta_{C}$ to the axis and azimuth $\alpha$, its velocity component toward an observer at inclination $i$ and angle $\phi$ is

$$
v(\alpha)=-v_{0}\left[\cos \theta_{C} \cos \gamma+\sin \theta_{C} \sin \gamma \cos \left(\alpha-\alpha_{0}\right)\right],
$$

where $\cos \gamma=\cos \phi \sin i$ and $\sin \phi \tan i \tan \alpha_{0}=1$. In axisymmetric flow $0 \leq \alpha \leq 2 \pi$ at fixed velocity, the mean observed velocity $\bar{v}=-v_{0} \cos \theta_{C} \cos \gamma$ is determined by the observer's orientation and naturally changes with binary phase. About $\pi / 2$ out of phase, so does the observed velocity width $v_{\max }-v_{\min }=$ $2 \sigma(v)=2 v_{0} \sin \theta_{C} \sin \gamma$ due to the azimuthal distribution of material. The geometry is sketched in Figure 11.

In the first Chandra observation, using the true anomaly of Table 2 to give $\cos \gamma_{1}=\cos \left(\nu_{1}-\omega-\pi\right) \sin i$, then $\tan \theta_{C} \tan \gamma_{1}=$ $\sigma\left(v_{1}\right) / \bar{v}_{1}$. With $\bar{v}_{1}=-600 \mathrm{~km} \mathrm{~s}^{-1}$ and $\mathrm{O}$ vIII's $\sigma\left(v_{1}\right)=850 \mathrm{~km}$ $\mathrm{s}^{-1}$ this implies $\theta_{C} \sim 54^{\circ}$ and $v_{0} \sim 1460 \mathrm{~km} \mathrm{~s}^{-1}$. A few days earlier, Varricatt et al. (2004) observed that the normally flattopped emission component of the He I $1.083 \mu \mathrm{m}$ line had developed a large extra blueshifted component at greater than X-ray velocities between about -50 and at least $-2200 \mathrm{~km} \mathrm{~s}^{-1}$ that they interpreted as arising from cool material farther out along the shock cone where the flow approached asymptotic conditions.

For the second Chandra observation, the only lines visible and resolved were those of $\mathrm{Si}$ and $\mathrm{S}$, whose wider velocity profiles reflect a contribution from the pressure-driven acceleration out of the system. Even so, if the flow pattern was the same in both observations, simple aspect scaling laws should apply. The low observed value of $\bar{v}_{2}=+159 \pm 167 \mathrm{~km} \mathrm{~s}^{-1}$ is consistent with the value $\bar{v}_{1} \cos \left(\nu_{2}-\omega-\pi\right) / \cos \left(\nu_{1}-\omega-\pi\right) \sim+185 \mathrm{~km} \mathrm{~s}^{-1}$ expected from scaling the observed pre-periastron Si XIV shift of $-660 \mathrm{~km} \mathrm{~s}^{-1}$ for the change of orientation between the two observations. Similarly, the complementary expectation that the line width should scale as $\sigma(v) \propto \sin (\nu-\omega-\pi)$ is also roughly borne out. We intend also to consider these questions of geometry in more detail in the forthcoming paper based on the RXTE monitoring program.

\subsection{The Nature of the Shock Transitions in WR 140}

A fundamental aspect of the flow of the stellar winds in WR 140 is that it involves converging charged-particle plasmas in which the collisions between particles on microscopic scales that collectively govern the macroscopic flow are due to long-distance Coulomb encounters as opposed to the familiar Earth-bound gas kinetic collisions of neutral particles. The structure of plasma shock waves to be expected in these circumstances has been discussed by Zel'dovich \& Raizer (2002, chap. 7, $\S 12)$. They emphasize the importance of two mechanisms: the slow character of Coulomb energy exchange between ions and electrons, and the effectiveness of electron heat conduction, at least in the absence of a dynamically significant magnetic field. Both of these aspects have been considered separately for models of WR 140 by Zhekov \& Skinner (2000) and Zhekov \& Myasnikov (2003), respectively, and are clearly fundamental in determining 


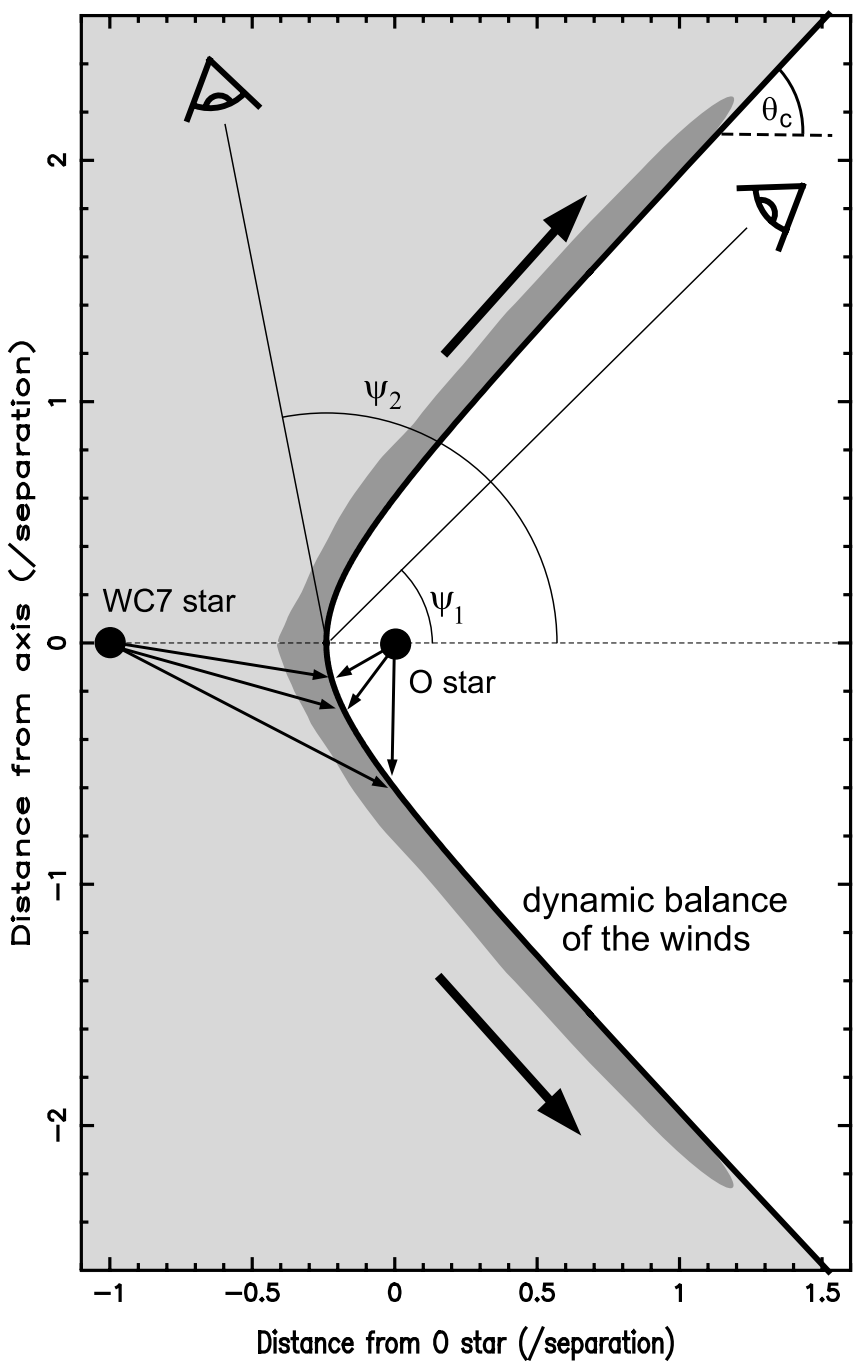

FIG. 11.-Sketch of the axisymmetric flow geometry assumed in WR 140 at the times of the two Chandra observations, in each case as a section through the plane defined by the axis joining the stars and the line of sight to the observer for an inclination of $60^{\circ}$. These planes are not perpendicular to the orbit. In order to parameterize the observed integrated velocity profiles, the form of the interaction region has been assumed to be the same at both epochs. It scales with the separation of the two stars and approximates at large distances to a cone of opening angle $\theta_{C}$ that scales with the separation of the two stars.

what is observed. As well as partly determining the energy balance of the postshock gas, shocked electrons are expected significantly to heat gas in otherwise cool preshock material by the conduction of heat from the shocked flow. A further consideration concerns the establishment of the shock discontinuities themselves.

In relaxation processes in general, the particle density is one of the quantities that determines the rate at which the postshock gas approaches equilibrium. The density calculated just before the shock surface closest to the stagnation point is a useful representative value. In convenient units, for a smooth, spherically symmetric wind in the terminal velocity regime, the ion density is $n_{i}(r)=1.34 \times 10^{8}\left(\dot{M}_{-6} / \mu v_{8}\right)(r / \mathrm{AU})^{-2} \mathrm{~cm}^{-3}$. At the stagnation point, $X$, in the WC star wind at $r \sim 0.8 D, n_{i}(X)=2.28 \times$ $10^{6}(D / a)^{-2} \mathrm{~cm}^{-3}$ and $n_{e} \sim 3.4 n_{i}$, where $(D / a)$ is again the binary separation in units of the semimajor axis. During the course of the eccentric binary orbit, $1-e \leq(D / a) \leq 1+e$, the stagnation point density, $n_{i}(X)$, changes by a factor of about 250 between $6.5 \times 10^{5}$ and $1.6 \times 10^{8} \mathrm{~cm}^{-3}$.
Judging by the Draine \& McKee (1993) criterion, the shocks in WR 140 are likely to be collisionless. The effective length for Coulomb collisional dissipation among ions following Spitzer (1962) is $l_{i-i} \sim 7.0 \times 10^{18} v_{8}^{4} / n_{i} \mathrm{~cm}$, a formula that applies to both protons and He nuclei. Calculated at the stagnation point

$$
l_{i-i} \sim 14(D / a)^{2} \mathrm{AU}
$$

so that even at the high densities of periastron $l_{i-i}$ is still about the expected width of the shocked region while at apastron it is larger than the binary separation. Under these circumstances, it is inevitable that dissipation occurs much more rapidly by the type of collective plasma processes that regulate the collisionless shocks that occur in the various astrophysical settings discussed by Draine \& McKee (1993).

The postshock electron temperature, $T_{e}$, is crucial in determining the X-ray spectrum. In a collisionless shock, electron heating is expected to be small, making $T_{e}$ lower than the ion temperature, $T_{i}$, although by how much is uncertain. Subsequently, equilibration takes place through Coulomb collisions between electrons and ions in a time

$$
t_{e-i} \sim 252\left(\mu / Z^{2}\right)(1 / \ln \Lambda)\left(T_{e}^{3 / 2} / n_{i}\right) \mathrm{s}
$$

(Spitzer 1962), where $\ln \Lambda$ is the Coulomb logarithm. For the postshock gas in WR 140, we can estimate an electron-ion relaxation length $l_{e-i}=t_{e-i} v_{\infty}$ by inserting the observed preperiastron electron continuum temperature from Table 9; the stagnation point density, $n_{i}(X)$; a value for the $\ln \Lambda=25$ from Spitzer (1962) appropriate for these densities and temperatures; and the observed WC abundances for $\mathrm{C} / \mathrm{He}=0.4$. With these approximations,

$$
l_{e-i} \sim 21(D / a)^{2} \mathrm{AU}
$$

which shows that, in the likely event of unequal postshock ion and electron temperatures, material will have flowed considerable distances downstream before temperature equilibrium can be established. This confirms the conclusions of Zhekov \& Skinner (2000), who used similar reasoning to find that simple nonequilibrium models gave a better fit to low-resolution $A S C A$ X-ray spectra.

Zel'dovich \& Raizer (2002) showed that the scale length of electron heat conduction is of the same order as $l_{e-i}$ so that this could speed up postshock equilibration. Whatever happens downstream, the emission lines in the WR 140 spectra show no evidence that heating by electron conduction took place in preshock gas. Any lines from this material would have the bulk high terminal wind velocities near $3000 \mathrm{~km} \mathrm{~s}^{-1}$ of the gas flowing into the shocks. In the pre-periastron spectrum, this would have been blueshifted from the WC star and redshifted from the O star but there was no sign of any emission of this type. The high-contrast bright Ne x line of Figure 6 might have been expected to be particularly sensitive to any such ionization precursor. It is probably the presence of a magnetic field that suppresses electron heat conduction into the preshock gas. WR 140's nonthermal radio emission, discussed below, shows that a field is certainly present and it is likely to be aligned along the shock surfaces, preventing conduction across the field lines.

The estimates of the Coulomb relaxation lengths of ions with ions or electrons with ions require values for $\ln \Lambda$, the Coulomb logarithm, that are usually taken from Spitzer's (1962) work. Recently, however, Li (2001) has argued that $\ln \Lambda$ should be 


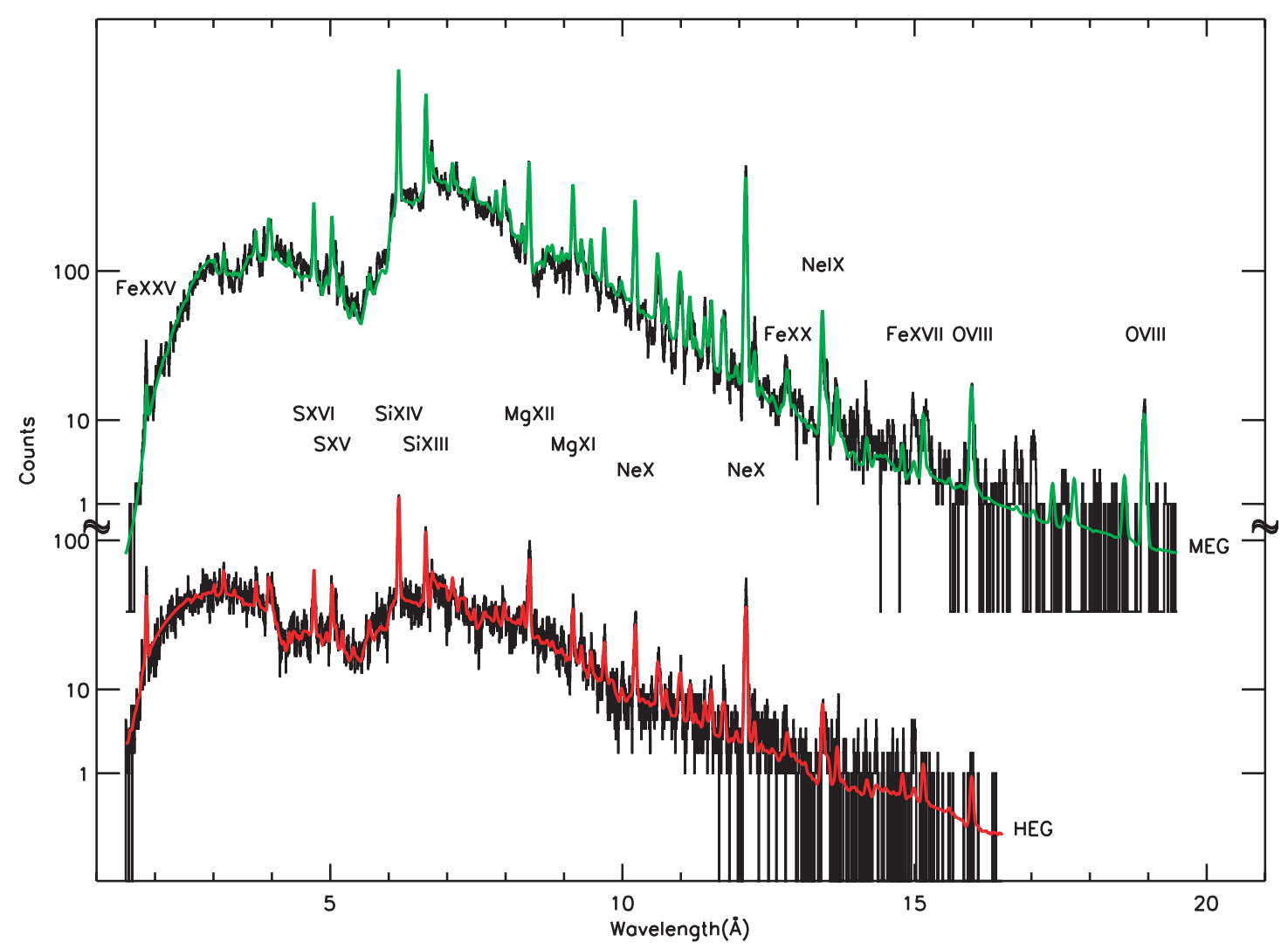

FIG. 12.-Comparison of the observed WR 140 Chandra MEG and HEG spectra with the best-fit XSPEC vpshock model subject to Gaussian smoothing. In order to emphasize both weak and strong lines, the intensity scale is the fourth root of the observed photon count.

reduced to half of its conventional value, which would increase proportionately the relaxation times.

Even as the electrons are heating up as gas flows out of the system, the ionization equilibrium remains to be established. According to Hughes \& Helfand (1985) or Hughes \& Singh (1994), this will have been completed by an ionization age $\tau=n_{e} t_{\text {ion }} \approx$ $10^{12.5} \mathrm{~cm}^{-3} \mathrm{~s}$, roughly independent of electron temperature (Masai 1994), with an associated length scale $l_{i o n}=t_{\text {ion }} v_{\infty}$ of

$$
l_{\text {ion }} \sim 8(D / a)^{2} \mathrm{AU}
$$

Thus, the postshock gas has to flow a significantly farther distance before the hottest ions are produced, although the observed ionization evolution of the outflowing gas primarily tracks the energy exchange between ions and electrons.

\subsection{WR 140 in Analogy with SNRs}

Nonthermal synchrotron radio emission is common in collisionless shocks observed from the Earth's magnetopause to SNRs, and so it is with WR 140 (White \& Becker 1995), which is bright and has a remarkable but unexplained dependence on binary phase. The Dougherty et al. (2005) imaging VLBA measurements imply prompt energy conversion at a shock front that is simultaneously responsible for both X-rays and radio. In this case, the jump conditions governing the shock structure need also to incorporate MHD terms and relativistic particle acceleration in addition to the ideal gas conditions that have hitherto been assumed exclusively to apply. These extra components to the energy budget are routine aspects of the dissipation in collisionless shocks that are likely to have observable consequences on WR 140's X-ray spectrum, perhaps most clearly in the unexpectedly low temperatures observed as reported in Table 9. The highest velocity observed in the resolved lines of about $2000 \mathrm{~km} \mathrm{~s}^{-1}$ is significantly lower than the values near $3000 \mathrm{~km} \mathrm{~s}^{-1}$ of the velocities of the preshock stellar winds but more consistent with the observed continuum temperature. This kinetic deficit may reflect the amount of energy dissipated by other means. The nonthermal particle population could help supply the inner-shell ionization proposed above to account for the strong forbiddenline emission among the He-like triplets.

Recent observations of young SNRs (e.g., Behar et al. 2001b) show that they share several of WR 140's characteristics including similar preshock gas velocities of a few thousand kilometers per second, simultaneous X-ray and nonthermal radio emission, a combination of apparently cool and hot lines, and much weaker intercombination than forbidden lines among the He-like triplets. SNRs generate shocked plasma out of ionization equilibrium as the blast wave sweeps through the ambient medium.

The nonequilibrium ionization models for the thermal X-ray spectra of SNRs developed by Borkowski et al. (2001) are available in XSPEC. The variable-abundance vpshock neivers 2.0 model subject to Gaussian smoothing for the line widths gives the excellent account shown in Figure 12 of the pre-periastron spectrum of WR 140 for the same data as Table 9, although we were not able to simulate an absence of hydrogen in the way we did above with the vmekal models. The relevant best-fit parameters, including the mean ionization age $\tau_{u}$, are given in Table 11. Both H-like and He-like lines are simultaneously reproduced quite well, although the forbidden lines look a bit weak, and nearly all of the Fe features are fine, except at the longest wavelengths. This success suggests that nonequilibrium processes are also at work in WR 140's stationary shocks. 
TABLE 11

Best-Fit vpshock Parameters of WR 140's Pre-Periastron X-RAy SPECTRUM

\begin{tabular}{|c|c|}
\hline Parameter & Value \\
\hline$N_{\mathrm{H}}\left(\mathrm{cm}^{-2}\right) \ldots \ldots \ldots$ & $(8.06 \pm 0.05) \times 10^{21}$ \\
\hline$k T(\mathrm{keV}) \ldots \ldots \ldots \ldots$ & $3.96 \pm 0.05$ \\
\hline$\tau_{u}\left(\mathrm{~s} \mathrm{~cm}^{-3}\right) \ldots \ldots$ & $(9.42 \pm 0.91) \times 10^{11}$ \\
\hline$C$-statistic.......... & 18208.7 \\
\hline
\end{tabular}

Far from earlier claims that high-resolution X-ray measurements of Wolf-Rayet stars would promise abundance measurements independent of ionization balance (e.g., Pollock 1995), for WR 140 exactly the opposite is true. Its emission-line spectrum will only be understood through a detailed hydrodynamic model incorporating collisionless shocks, mediated to an extent yet to be understood by nonthermal particle acceleration, and subsequent postshock evolution of the electron temperature and ion population.

The Chandra observations were eventually awarded under Director's Discretionary time for which we are extremely grateful. We are also delighted to acknowledge extensive use of the APED ${ }^{6}$ and $\mathrm{CHIANTI}^{7}$ atomic databases. Dave Henley kindly supplied Figure 2 and read the article with care. Thanks are also due to the anonymous referee, whose painstaking reports called our attention to a number of things that improved the paper considerably.

\footnotetext{
6 See http://cxc.harvard.edu/atomdb/index.html.

7 CHIANTI is a collaborative project involving the NRL (US), RAL (UK), and the Universities of Florence (Italy) and Cambridge (UK).
}

Behar, E., Cottam, J., \& Kahn, S. M. 2001a, ApJ, 548, 966

Behar, E., Rasmussen, A. P., Griffiths, R. G., Dennerl, K., Audard, M., Aschenbach, B., \& Brinkman, A. C. 2001b, A\&A, 365, L242

Berghöfer, T. W., Schmitt, J. H. M. M., \& Cassinelli, J. P. 1996, A\&AS, 118, 481

Borkowski, K. J., Lyerly, W. J., \& Reynolds, S. P. 2001, ApJ, 548, 820

Brown, G. V., Beiersdorfer, P., Liedahl, D. A., Widmann, K., Kahn, S. M., \& Clothiaux, E. J. 2002, ApJS, 140, 589

Corcoran, M. F., et al. 2001, ApJ, 562, 1031

Decaux, V., Beiersdorfer, P., Kahn, S. M., \& Jacobs, V. L. 1997, ApJ, 482, 1076

Dessart, L., Crowther, P. A., Hillier, J. D., Willis, A. J., Morris, P. W., \& van der Hucht, K. A. 2000, MNRAS, 315, 407

Dougherty, S. M., Beasley, A. J., Claussen, M. J., Zauderer, B. A., \& Bolingbroke, N. J. 2005, ApJ, 623, 447

Draine, B. T., \& McKee, C. F. 1993, ARA\&A, 31, 373

Eenens, P. R. J., \& Williams, P. M. 1992, MNRAS, 255, 227

Gräfener, G., Hamann, W.-R., Hillier, D. J., \& Koesterke, L. 1998, A\&A, 329, 190

Henley, D. B., Stevens, I. R., \& Pittard, J. M. 2003, MNRAS, 346, 773

Hillier, D. J., \& Miller, D. L. 1999, ApJ, 519, 354

Hughes, J. P., \& Helfand, D. J. 1985, ApJ, 291, 544

Hughes, J. P., \& Singh, K. P. 1994, ApJ, 422, 126

Li, D. 2001, Nucl. Fusion, 41, 631

Lührs, S. 1997, PASP, 109, 504

Maeda, Y., Koyama, K., Yokogawa, J., \& Skinner, S. 1999, ApJ, 510, 967

Marchenko, S. V., et al. 2003, ApJ, 596, 1295

Masai, K. 1994, ApJ, 437, 770

McKenzie, D. L., Landecker, P. B., Broussard, R. M., Rugge, H. R., Young, R. M., Feldman, U., \& Doschek, G. A. 1980, ApJ, 241, 409

Mewe, R., \& Schrijver, J. 1978, A\&A, 65, 115

Miller, N. A., Cassinelli, J. P., Waldron, W. L., MacFarlane, J. J., \& Cohen, D. H. 2002, ApJ, 577, 951

Monnier, J. D., Tuthill, P. G., \& Danchi, W. C. 2002, ApJ, 567, L137

Monnier, J. D., et al. 2004, ApJ, 602, L57

Morris, P. W., Brownsberger, K. R., Conti, P. S., Massey, P., \& Vacca, W. D. 1993, ApJ, 412, 324

Ness, J.-U., Schmitt, J. H. M. M., Burwitz, V., Mewe, R., Raassen, A. J. J., van der Meer, R. L. J., Predehl, P., \& Brinkman, A. C. 2002, A\&A, 394, 911

Oskinova, L. M., Ignace, R., Hamann, W.-R., Pollock, A. M. T., \& Brown, J. C. 2003, A\&A, 402, 755

Pittard, J. M., \& Stevens, I. R. 2002, A\&A, 388, L20

\section{EFERENCES}

Pollock, A. M. T. 1987, A\&A, 171, 135

1995, in IAU Symp. 163, Wolf-Rayet Stars: Binaries, Colliding Winds, Evolution, ed. K. A. van der Hucht \& P. M. Williams (Dordrecht: Kluwer), 429

Pollock, A. M. T., Corcoran, M. F., \& Stevens, I. R. 2002, in ASP Conf. Ser. 260, Interacting Winds from Massive Stars, ed. A. F. J. Moffat \& N. St-Louis (San Francisco: ASP), 537

Porquet, D., Mewe, R., Dubau, J., Raassen, A. J. J., \& Kaastra, J. S. 2001, A\&A, 376, 1113

Pradhan, A. K. 1982, ApJ, 263, 477

Schulz, N. S., Canizares, C., Huenemoerder, D., \& Tibbets, K. 2003, ApJ, 595, 365 Setia Gunawan, D. Y. A., van der Hucht, K. A., Williams, P. M., Henrichs, H. F., Kaper, L., Stickland, D. J., \& Wamsteker, W. 2001, A\&A, 376, 460

Skinner, S. L., Güdel, M., Schmutz, W., \& Stevens, I. R. 2001, ApJ, 558, L113 Smith, R. K., Brickhouse, N. S., Liedahl, D. A., \& Raymond, J. C. 2001, ApJ, 556, L91

Spitzer, L., Jr. 1962, Physics of Fully Ionized Gases (2nd ed.; New York: Wiley)

Stevens, I. R., Blondin, J. M., \& Pollock, A. M. T. 1992, ApJ, 386, 265

Uyaniker, B., Fürst, E., Reich, W., Aschenbach, B., \& Wielebinski, R. 2001, A\&A, 371, 675

van der Hucht, K. A. 2001, NewA Rev., 45, 135

van der Hucht, K. A., Cassinelli, J. P., \& Williams, P. M. 1986, A\&A, 168, 111 Varricatt, W. P., Williams, P. M., \& Ashock, N. M. 2004, MNRAS, 351, 1307

Vuong, M. H., Montmerle, T., Grosso, N., Feigelson, E. D., Verstraete, L., \& Ozawa, H. 2003, A\&A, 408, 581

Weisskopf, M. C., Brinkman, B., Canizares, C., Garmire, G., Murray, S., \& van Speybroeck, L. P. 2002, PASP, 114, 1

White, R. L., \& Becker, R. H. 1995, ApJ, 451, 352

Williams, P. M., van der Hucht, K. A., Pollock, A. M. T, Florkowski, D. R., van der Woerd, H., \& Wamsteker, W. M. 1990, MNRAS, 243, 662

Wolfson, C. J., Leibacher, J. W., Doyle, J. G., \& Phillips, K. J. H. 1983, ApJ, 269,319

Young, P. R., Del Zanna, G., Landi, E., Dere, K. P., Mason, H. E., \& Landini, M. 2003, ApJS, 144, 135

Zel'dovich, Ya. B., \& Raizer, Yu. P. 2002, Physics of Shock Waves and HighTemperature Hydrodynamic Phenomena (Mineola: Dover)

Zhekov, S. A., \& Myasnikov, A. V. 2003, Astron. Lett., 29, 394

Zhekov, S. A., \& Skinner, S. L. 2000, ApJ, 538, 808 